\title{
Site-Specific Prodrug Release Using Visible Light
}

\author{
Michael Y. Jiang and David Dolphin* \\ Supporting Information
}

\section{Instrumentation and General Materials}

The NMR spectra were recorded on a Bruker AV-300 or a Bruker AV-400 spectrometer in the solvents indicated and were referenced to residual solvent peaks. Low and high resolution mass spectra were determined on a Micromass LCT TOF-ESI mass spectrometer or a KRATOS Concept IIHQ hybrid mass spectrometer. Elemental analyses were performed on a Carlo Erba Elemental Analyzer 1108. The UV-Vis spectra were recorded on a Varian Cary-50 spectrophotometer. Gas chromatography (GC) was carried out on a HP 5890 instrument equipped with a flame ionization detector (FID) and a HP17 column. Photooxygenation experiments were carried out with a $250 \mathrm{~W}$ (maximum) Osram HLX 64655 arc lamp in an Oriel lamp housing (model 66184) and the light output passed through a $\mathrm{K}_{2} \mathrm{Cr}_{2} \mathrm{O}_{7}$ aq. filter solution $(0.2 \mathrm{M}, \lambda>550 \mathrm{~nm})$ or a Corion P70-650-S filter $(680 \mathrm{~nm}>\lambda>620 \mathrm{~nm})$.

All chemicals were purchased from Sigma-Aldrich, Acros or Fisher Scientific and purified by published procedures if necessary. ${ }^{1}$ Ring B-BPD- $7^{2}$-monoacid (BPDA) was obtained from QLT Inc. Deuterated solvents were purchased from Cambridge Isotope Laboratories or Aldrich. Flash chromatography was performed on silica gel (Silicycle or BDH, 70-230 / 230-400 mesh) and basic alumina (Brockman, 60-325 mesh, deactivated with $3 \%$ water to activity II or $6 \%$ water to activity III). Analytical Thin 
Layer Chromatography (TLC) was performed on aluminum backed plates pre-coated with silica (Merck or Aldrich, $0.2 \mathrm{~mm}, 60$ mesh with F254 indicator) or alumina (Merck, $0.2 \mathrm{~mm}$, activity III, 60 mesh with F254 indicator). Preparative TLC was performed on glass backed plates pre-coated with silica (Merck, $0.5 \mathrm{~mm}$ and $1 \mathrm{~mm}, 60$ mesh without indicator).

\section{Synthesis}

\section{(5-Hydroxypentoxy)(tert-butyl)dimethylsilane}

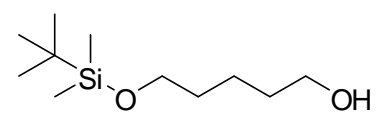

To a $100 \mathrm{~mL}$ dry THF suspension of $\mathrm{NaH}$ ( $3.9 \mathrm{~g}, 60 \%$ suspension in mineral oil, $99 \mathrm{mmol})$ at $0{ }^{\circ} \mathrm{C}$ was added dropwise a THF solution $(20 \mathrm{~mL})$ of 1,5-pentanediol $(9.5$ $\mathrm{mL}, 90 \mathrm{mmol})$, followed by the addition of a THF solution $(30 \mathrm{~mL})$ of tertbutyldimethylsilyl chloride (13.6 g, $90 \mathrm{mmol})$. The solution was stirred overnight before being quenched with sat. $\mathrm{NaHCO}_{3}$ aq. solution and extracted with ether. The organic phase was concentrated and chromatographed on silica gel (ethyl acetate:hexane $=1: 4$ ) to give the title product ( $12.2 \mathrm{~g}, 62 \%$ yield).

$$
\left.\mathrm{R}_{f} \text { (silica-ethyl acetate:hexane }=1: 2\right): 0.4 ;{ }^{1} \mathrm{H} \text { NMR }\left(400 \mathrm{MHz}, \mathrm{CDCl}_{3}\right): \delta=3.56-
$$

$3.52(\mathrm{~m}, 4 \mathrm{H}), 2.56-2.48$ (br. $\mathrm{s}, 1 \mathrm{H}), 1.52-1.44(\mathrm{~m}, 4 \mathrm{H}), 1.36-1.30(\mathrm{~m}, 2 \mathrm{H}), 0.82(\mathrm{~s}, 9 \mathrm{H}),-$

$0.02(\mathrm{~s}, 6 \mathrm{H}) ;{ }^{13} \mathrm{C}\left[{ }^{1} \mathrm{H}\right] \mathrm{NMR}\left(100 \mathrm{MHz}, \mathrm{CDCl}_{3}\right): \delta=63.1,62.5,32.4,32.3,25.8,21.9$, 18.2, -5.4; LRESIMS (m/e): $241.4\left([\mathrm{M}+\mathrm{Na}]^{+}\right)$; HRESIMS (m/e): calcd. for $\mathrm{C}_{11} \mathrm{H}_{26} \mathrm{O}_{2} \mathrm{NaSi}$ $\left([\mathrm{M}+\mathrm{Na}]^{+}\right) 241.1600$, found 241.1595. These data agree with the literature data. ${ }^{2}$ 


\section{[Bis(phenylthio)methyl]tributylstannane}

$$
\mathrm{Bu}_{3} \mathrm{Sn} \overbrace{\mathrm{SPh}}^{\mathrm{SPh}}
$$

To a dry THF solution $(20 \mathrm{~mL})$ of bis(phenylthio)methane $(2.32 \mathrm{~g}, 10 \mathrm{mmol})$ at $78{ }^{\circ} \mathrm{C}$ under Ar was added dropwise a LDA solution $(5.5 \mathrm{~mL}, 2 \mathrm{M}$ solution in heptane/THF/ethylbenzene, $11 \mathrm{mmol}$ ). The solution was stirred for $1 \mathrm{~h}$ before the addition of a THF solution $(10 \mathrm{~mL})$ of tributyltin chloride $(3 \mathrm{~mL}, 11 \mathrm{mmol})$ and stirring was continued overnight. The reaction mixture was quenched with water, extracted with hexane and dried over sodium sulfate. The solution was then concentrated and chromatographed on silica gel (hexane) to give the title product ( $5 \mathrm{~g}, 95 \%$ yield).

$\mathrm{R}_{f}$ (silica- hexane): 0.16; ${ }^{1} \mathrm{H}$ NMR $\left(400 \mathrm{MHz}, \mathrm{CDCl}_{3}\right): \delta=7.43-7.41(\mathrm{~m}, 4 \mathrm{H})$, 7.21-7.16 (m, 6H), $4.31(\mathrm{~s}, 1 \mathrm{H}), 1.54-1.49(\mathrm{~m}, 6 \mathrm{H}), 1.35-1.27(\mathrm{~m}, 6 \mathrm{H}), 1.01-0.97(\mathrm{~m}, 6 \mathrm{H})$, $0.91(\mathrm{t}, 9 \mathrm{H}, \mathrm{J}=7.3 \mathrm{~Hz}) ;{ }^{13} \mathrm{C}\left[{ }^{1} \mathrm{H}\right] \mathrm{NMR}\left(100 \mathrm{MHz}, \mathrm{CDCl}_{3}\right): \delta=137.6,130.9,130.8,128.6$, 126.7, 36.4, 36.3, 28.8, 27.3, 13.6, 11.0; LRESIMS (m/e): $544.9\left([\mathrm{M}+\mathrm{Na}]^{+}\right)$; HRESIMS (m/e): calcd. for $\mathrm{C}_{25} \mathrm{H}_{38} \mathrm{~S}_{2} \mathrm{SnNa}\left([\mathrm{M}+\mathrm{Na}]^{+}\right)$545.1335, found 545.1344; Anal. calcd. (found) for $\mathrm{C}_{25} \mathrm{H}_{38} \mathrm{~S}_{2} \mathrm{Sn}$ : C, 57.59 (57.80); H, 7.35 (7.60).

\section{[5-(Bisphenylthiomethoxy)pentyloxy](tert-butyl)dimethylsilane $\mathbf{1}$}

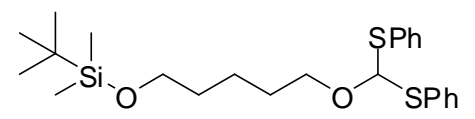

Using a modified literature procedure, ${ }^{3}$ to a $100 \mathrm{~mL}$ dry THF suspension of $\mathrm{LiBr}$ (4.3 g, $50 \mathrm{mmol})$ and (5-hydroxypentoxy)tert-butyldimethylsilane (5.5 g, $25 \mathrm{mmol})$ at 0 ${ }^{\circ} \mathrm{C}$ under $\mathrm{Ar}$ was added dropwise n-butyllithium solution $(16 \mathrm{~mL}, 1.6 \mathrm{M}$ solution in hexane, $25 \mathrm{mmol}$ ) and the reaction mixture was stirred for $30 \mathrm{~min}$, before it was cooled to 
$-40{ }^{\circ} \mathrm{C}$ and $\mathrm{CuBr}_{2}(12.3 \mathrm{~g}, 55 \mathrm{mmol})$ was then added. Stirring was continued for $30 \mathrm{~min}$ before a THF solution $(100 \mathrm{~mL})$ of (bis(phenylthio)methyl)tributylstannane (13.1 g, 25 mmol) was added. The reaction mixture was stirred overnight while slowly warming to $25{ }^{\circ} \mathrm{C}$, before it was quenched with $3.5 \%$ ammonium hydroxide aq. solution and the insoluble materials were filtered off. The filtrate was then concentrated and chromatographed on silica gel $\left(\mathrm{CH}_{2} \mathrm{Cl}_{2}\right.$ :hexane $\left.=1: 1\right)$ to give $\mathbf{1}(6.5 \mathrm{~g}, 58 \%$ yield $)$.

$$
\left.\mathrm{R}_{f} \text { (silica- } \mathrm{CH}_{2} \mathrm{Cl}_{2} \text { :hexane }=1: 4\right): 0.15 ;{ }^{1} \mathrm{H} \text { NMR }\left(400 \mathrm{MHz}, \mathrm{C}_{6} \mathrm{D}_{6}\right): \delta=7.50-7.47
$$

(m, 4H), 7.03-6.94 (m, 6H), $6.27(\mathrm{~s}, 1 \mathrm{H}), 3.72(\mathrm{t}, 2 \mathrm{H}, \mathrm{J}=6.4 \mathrm{~Hz}), 3.42(\mathrm{t}, 2 \mathrm{H}, \mathrm{J}=6.3 \mathrm{~Hz})$,

1.45-1.33 (m, 4H), 1.27-1.21 (m, 2H), 0.98 (s, 9H), $0.05(\mathrm{~s}, 6 \mathrm{H}) ;{ }^{13} \mathrm{C}\left[{ }^{1} \mathrm{H}\right]$ NMR (100 $\left.\mathrm{MHz}, \mathrm{C}_{6} \mathrm{D}_{6}\right): \delta=135.1,133.0,129.5,128.2,94.0,66.4,63.4,33.1,29.6,26.5,23.1,18.9$, -5.38; LRESIMS (m/e): $471.2\left([\mathrm{M}+\mathrm{Na}]^{+}\right)$; HRESIMS (m/e): calcd. for $\mathrm{C}_{24} \mathrm{H}_{36} \mathrm{O}_{2} \mathrm{~S}_{2} \mathrm{SiNa}$ $\left([\mathrm{M}+\mathrm{Na}]^{+}\right)$471.1824, found 471.1842; Anal. calcd. (found) for $\mathrm{C}_{24} \mathrm{H}_{36} \mathrm{O}_{2} \mathrm{~S}_{2} \mathrm{Si}$ : C, 64.23 (64.42); H, 8.09 (7.93).

Methyl 2-(4-isobutylphenyl)propanoate (methyl ester of ibuprofen)

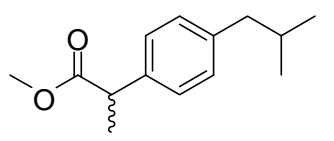

To a methanol solution $(30 \mathrm{~mL})$ of 2-(4-isobutylphenyl)propanoic acid (ibuprofen, $0.5 \mathrm{~g}, 2.4 \mathrm{mmol}$ ) was added 3 drops of conc. $\mathrm{H}_{2} \mathrm{SO}_{4}$ and the reaction mixture was stirred for $4 \mathrm{~h}$ before it was diluted with $\mathrm{CH}_{2} \mathrm{Cl}_{2}$, washed with sat. $\mathrm{NaHCO}_{3}$ aq. solution and dried over sodium sulfate. Solvent evaporation gave the title methyl ester quantitatively $(0.53 \mathrm{~g})$.

${ }^{1} \mathrm{H}$ NMR (400 MHz, $\left.\mathrm{C}_{6} \mathrm{D}_{6}\right): \delta=7.24(\mathrm{~d}, 2 \mathrm{H}, \mathrm{J}=8.1 \mathrm{~Hz}), 6.98(\mathrm{~d}, 2 \mathrm{H}, \mathrm{J}=8.0 \mathrm{~Hz})$, $3.57(\mathrm{q}, 1 \mathrm{H}, \mathrm{J}=7.1 \mathrm{~Hz}), 3.25(\mathrm{~s}, 3 \mathrm{H}), 2.29(\mathrm{~d}, 2 \mathrm{H}, \mathrm{J}=7.2 \mathrm{~Hz}), 1.74-1.67(\mathrm{~m}, 1 \mathrm{H}), 1.42$ 
$(\mathrm{d}, 3 \mathrm{H}, \mathrm{J}=7.1 \mathrm{~Hz}), 0.80(\mathrm{~d}, 6 \mathrm{H}, \mathrm{J}=6.6 \mathrm{~Hz}) ;{ }^{13} \mathrm{C}\left[{ }^{1} \mathrm{H}\right] \mathrm{NMR}\left(100 \mathrm{MHz}, \mathrm{C}_{6} \mathrm{D}_{6}\right): \delta=174.9$, 141.0, 139.0, 130.0, 128.0, 51.7, 45.7, 45.6, 30.8, 22.8, 19.3; LRESIMS (m/e): 243.2 $\left([\mathrm{M}+\mathrm{Na}]^{+}\right)$; HRESIMS (m/e): calcd. for $\mathrm{C}_{14} \mathrm{H}_{20} \mathrm{O}_{2} \mathrm{Na}\left([\mathrm{M}+\mathrm{Na}]^{+}\right)$243.1361, found 243.1360. These data are consistent with the literature data. ${ }^{4}$

(S)-Methyl 2-(6-methoxynaphthalen-2-yl)propanoate (methyl ester of naproxen)

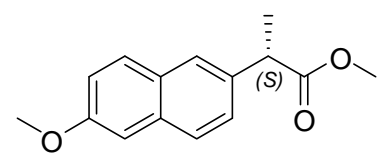

Following the preparation procedure for ibuprofen methyl ester, (S)-2-(6 methoxynaphthalen-2-yl)propanoic acid (naproxen, $0.5 \mathrm{~g}, 2.2 \mathrm{mmol}$ ) was converted to the title methyl ester quantitatively $(0.54 \mathrm{~g})$.

${ }^{1} \mathrm{H}$ NMR $\left(400 \mathrm{MHz}, \mathrm{CDCl}_{3}\right): \delta=7.62(\mathrm{~s}, 1 \mathrm{H}), 7.57(\mathrm{~d}, 1 \mathrm{H}, \mathrm{J}=8.5 \mathrm{~Hz}), 7.48-$ $7.45(\mathrm{~m}, 2 \mathrm{H}), 7.18(\mathrm{~d}, 1 \mathrm{H}, \mathrm{J}=2.6 \mathrm{~Hz}), 6.89(\mathrm{~d}, 1 \mathrm{H}, \mathrm{J}=2.4 \mathrm{~Hz}), 3.71(\mathrm{q}, 1 \mathrm{H}, \mathrm{J}=7.1 \mathrm{~Hz})$ $3.36(\mathrm{~s}, 3 \mathrm{H}), 3.28(\mathrm{~s}, 3 \mathrm{H}), 1.52(\mathrm{~d}, 3 \mathrm{H}, \mathrm{J}=7.1 \mathrm{~Hz}) ; \operatorname{LRESIMS}(\mathrm{m} / \mathrm{e}): 267.0\left([\mathrm{M}+\mathrm{Na}]^{+}\right)$; HRESIMS (m/e): calcd. for $\mathrm{C}_{15} \mathrm{H}_{16} \mathrm{O}_{3} \mathrm{Na}\left([\mathrm{M}+\mathrm{Na}]^{+}\right)$267.0997, found 267.0999. These data are consistent with the literature data. ${ }^{5}$

\section{5-(4-Methoxycarbonylphenyl)-10,15,20-triphenylporphyrin}

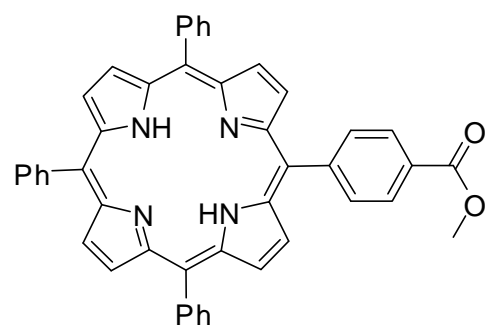

Following the literature procedure, ${ }^{6}$ to a stirred degassed $\mathrm{CHCl}_{3}$ solution $(800$ 
$\mathrm{mL}$ ) of benzaldehyde (3.7 mL, $37 \mathrm{mmol})$, methyl 4-formylbenzoate (2.0 g, $12.3 \mathrm{mmol})$ and pyrrole $(3.1 \mathrm{~mL}, 44.5 \mathrm{mmol})$ was added $\mathrm{BF}_{3} \cdot \mathrm{OEt}_{2}(0.2 \mathrm{~mL}, 1.6 \mathrm{mmol})$ dropwise. Stirring was continued for $19 \mathrm{~h}$ under Ar before the addition of DDQ (5.1 g, $22.3 \mathrm{mmol})$. The mixture was maintained at $r$. t. for 1 day before solvent evaporation and purification by silica gel chromatography $\left(\mathrm{CH}_{2} \mathrm{Cl}_{2}\right.$ :hexane $=2: 3$ as eluant $)$ to give the title compound $(1.217 \mathrm{~g}, 20 \%)$

$\mathrm{R}_{f}\left(\right.$ silica- $\left.\mathrm{CH}_{2} \mathrm{Cl}_{2}\right): 0.53 ;{ }^{1} \mathrm{H}$ NMR $\left(400 \mathrm{MHz}, \mathrm{CDCl}_{3}\right): \delta=8.86-8.84(\mathrm{~m}, 6 \mathrm{H})$, $8.78(\mathrm{~d}, 2 \mathrm{H}, \mathrm{J}=4.8 \mathrm{~Hz}), 8.43(\mathrm{~d}, 2 \mathrm{H}, \mathrm{J}=8.2 \mathrm{~Hz}), 8.30(\mathrm{~d}, 2 \mathrm{H}, \mathrm{J}=8.2 \mathrm{~Hz}), 8.22-8.20(\mathrm{~m}$, 6H), 7.79-7.72 (m, 9H), 4.10 (s, 3H), -2.64 (br. s, 2H); LRESIMS (m/e): $673.4\left([\mathrm{M}+\mathrm{H}]^{+}\right)$; HRESIMS (m/e): calcd. for $\mathrm{C}_{46} \mathrm{H}_{33} \mathrm{~N}_{4} \mathrm{O}_{2}\left([\mathrm{M}+\mathrm{H}]^{+}\right)$673.2604, found 673.2601; UV-Vis $\left(\mathrm{CH}_{2} \mathrm{Cl}_{2}\right) \lambda_{\max } / \mathrm{nm}: 418.9,515.0,550.1,591.0,646.0$. These data agree with the literature data. ${ }^{6}$

5-(4-Carboxyphenyl)-10,15,20-triphenylporphyrin (TPPA)

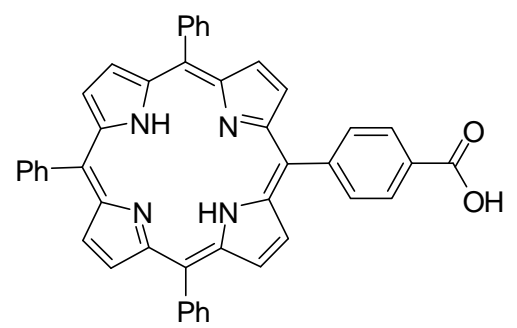

Following the literature procedure, ${ }^{7}$ to a solution of 5-(4methoxycarbonylphenyl)-10,15,20-triphenylporphyrin (1.22 g, $1.82 \mathrm{mmol})$ in EtOH (60 $\mathrm{mL}$ ) was added $2 \mathrm{M}$ aq. $\mathrm{KOH}$ solution $(120 \mathrm{~mL})$ and the suspension was refluxed overnight. After cooling to r. t., the solvent was decanted and the crude mixture was washed with water, $\mathrm{MeOH}$ and hexane then evaporated to dryness. The residue was preadsorbed onto silica and chromatographed (silica, $5 \% \mathrm{MeOH}$ in $\mathrm{CH}_{2} \mathrm{Cl}_{2}$ ) to give the title 
compound $(0.85 \mathrm{~g}, 71 \%)$.

$\mathrm{R}_{f}$ (silica-5\% MeOH in $\mathrm{CH}_{2} \mathrm{Cl}_{2}$ ): 0.24; ${ }^{1} \mathrm{H}$ NMR (400 MHz, $\mathrm{d}_{6}$-acetone): $\delta=$ 8.89-8.87 (m, 8H), 8.49 (d, 2H, J = 8.0 Hz), 8.39 (d, 2H, J = 8.2 Hz), 8.27-8.24 (m, 6H), 7.86-7.82 (m, 9H), -2.75 (s, 2H); ESIMS (m/e): $659.1\left([\mathrm{M}+\mathrm{H}]^{\dagger}\right)$; HRESIMS (m/e): calcd. for $\mathrm{C}_{45} \mathrm{H}_{31} \mathrm{~N}_{4} \mathrm{O}_{2}\left([\mathrm{M}+\mathrm{H}]^{+}\right)$659.2447, found 659.2467. These data are consistent with the literature data. ${ }^{7}$

\section{General Procedure for Takeda Alkoxymethylenation ${ }^{3}$ (GP-1)}

$\mathrm{Mg}$ turnings $(86.4 \mathrm{mg}, 3.6 \mathrm{mmol}), \mathrm{Cp}_{2} \mathrm{TiCl}_{2}(0.75 \mathrm{~g}, 3 \mathrm{mmol})$ and finely powdered 4 Å molecular sieves $(300 \mathrm{mg}$ ) were dried by heating in vacuo. After cooling under Ar, dry THF $(5 \mathrm{~mL})$ and $\mathrm{P}(\mathrm{OEt})_{3}(1.05 \mathrm{~mL}, 6 \mathrm{mmol})$ were added to the system and the solution was stirred at $25^{\circ} \mathrm{C}$ for $3 \mathrm{~h}$, before the addition of a THF solution $(1.5 \mathrm{~mL})$ of $1(0.34 \mathrm{~g}, 0.75 \mathrm{mmol})$ and stirring was continued for $15 \mathrm{~min}$. A THF solution $(2.5 \mathrm{~mL})$ of ester or amide substrate $(0.5 \mathrm{mmol})$ was then added dropwise over $10 \mathrm{~min}$ to the system and stirring was continued for another $3 \mathrm{~h}$. The reaction was quenched with $1 \mathrm{M}$ aq. $\mathrm{NaOH}$ solution and the insoluble materials were filtered off through Celite and washed with ether. The layers were separated and the aq. layer was extracted with ether. The combined organic extracts were dried over sodium sulfate before they were concentrated and chromatographed on basic alumina (activity (II)) to furnish enol ether products. In cases where the complete separation of Z- and E- product isomers was difficult, the diastereomer mixture was carried forward to the next step.

\section{$\underline{\text { 2,2,3,3-Tetramethyl-12-propyl-4,10,13-trioxa-3-silapentadec-11-ene }}$}




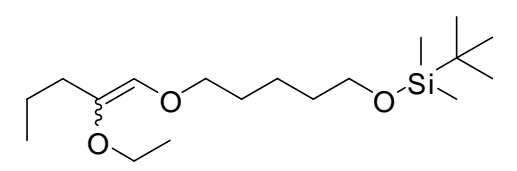

Following the general procedure (GP-1), ethyl butyrate ( $71 \mu \mathrm{L}, 0.5 \mathrm{mmol})$ was alkoxymethylenated. The resulting crude mixture was concentrated and chromatographed on basic alumina (activity (II), $\mathrm{CH}_{2} \mathrm{Cl}_{2}$ :hexane $=1: 5$ ) to give three fractions: Z-isomer (4 $\mathrm{mg}, 2 \%$ yield), a Z/E mixture 1 (54 $\mathrm{mg}, 33 \%$ yield, $Z: E=17: 83)$ and a $\mathrm{Z} / \mathrm{E}$ mixture 2 (33 $\mathrm{mg}, 20 \%$ yield, $\mathrm{Z}: \mathrm{E}=71: 29)$. The combined yield was $55 \%$ with a stereoselectivity of $\mathrm{Z}: \mathrm{E}=39: 61$.

\section{(Z)-Isomer}

$\mathrm{R}_{f}\left(\right.$ silica- $\mathrm{CH}_{2} \mathrm{Cl}_{2}$ ): 0.28; ${ }^{1} \mathrm{H}$ NMR (400 MHz, $\left.\mathrm{C}_{6} \mathrm{D}_{6}\right): \delta=5.29(\mathrm{~s}, 1 \mathrm{H}), 4.17$ (q, $2 \mathrm{H}, \mathrm{J}=7.0 \mathrm{~Hz}), 3.51(\mathrm{t}, 2 \mathrm{H}, \mathrm{J}=6.1 \mathrm{~Hz}), 3.43(\mathrm{t}, 2 \mathrm{H}, \mathrm{J}=6.3 \mathrm{~Hz}), 1.92(\mathrm{t}, 2 \mathrm{H}, \mathrm{J}=7.2 \mathrm{~Hz})$, 1.61-1.35 (m, 8H), $1.25(\mathrm{t}, 3 \mathrm{H}, \mathrm{J}=7.0 \mathrm{~Hz}), 0.99(\mathrm{~s}, 9 \mathrm{H}), 0.93(\mathrm{t}, 3 \mathrm{H}, \mathrm{J}=7.4 \mathrm{~Hz}), 0.06(\mathrm{~s}$, $6 \mathrm{H}) ;{ }^{13} \mathrm{C}\left[{ }^{1} \mathrm{H}\right] \mathrm{NMR}\left(100 \mathrm{MHz}, \mathrm{C}_{6} \mathrm{D}_{6}\right): \delta=138.4,127.8,72.2,65.6,62.8,33.5,32.5,29.5$, 25.8, 22.4, 20.7, 18.1, 15.7, 13.4, -5.5; LRESIMS (m/e): $353.3\left([\mathrm{M}+\mathrm{Na}]^{+}\right)$.

\section{2,2,3,3-Tetramethyl-12-phenyl-4,10,13-trioxa-3-silapentadec-11-ene}

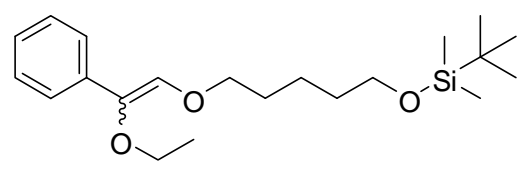

Following the general procedure (GP-1), ethyl benzoate ( $72 \mu \mathrm{L}, 0.5 \mathrm{mmol}$ ) was alkoxymethylenated. The resulting crude mixture was concentrated and chromatographed on basic alumina (activity (II), $\mathrm{CH}_{2} \mathrm{Cl}_{2}$ :hexane $=1: 6$ as eluant) to give two fractions: $\mathrm{Z}$ isomer (24.9 $\mathrm{mg}, 14 \%$ yield) and a Z/E mixture ( $43.6 \mathrm{mg}, 24 \%$ yield, $\mathrm{Z}: \mathrm{E}=86: 14)$. The combined yield was $38 \%$ with a stereoselectivity of $Z: E=91: 9$. 
(Z)-Isomer

$\mathrm{R}_{f}\left(\right.$ silica- $\left.\mathrm{CH}_{2} \mathrm{Cl}_{2}\right): 0.45 ;{ }^{1} \mathrm{H}$ NMR $\left(400 \mathrm{MHz}, \mathrm{C}_{6} \mathrm{D}_{6}\right): \delta=7.51(\mathrm{~d}, 2 \mathrm{H}, \mathrm{J}=8.5$ Hz), 7.21-7.17 (m, 2H), 7.10-7.07 (m, 1H), $6.13(\mathrm{~s}, 1 \mathrm{H}), 4.03$ (q, 2H, J = 7.0 Hz), 3.51$3.47(\mathrm{~m}, 4 \mathrm{H}), 1.47-1.35(\mathrm{~m}, 6 \mathrm{H}), 1.27(\mathrm{t}, 3 \mathrm{H}, \mathrm{J}=7.0 \mathrm{~Hz}), 0.99(\mathrm{~s}, 9 \mathrm{H}), 0.06(\mathrm{~s}, 6 \mathrm{H})$; ${ }^{13} \mathrm{C}\left[{ }^{1} \mathrm{H}\right] \mathrm{NMR}\left(100 \mathrm{MHz}, \mathrm{C}_{6} \mathrm{D}_{6}\right): \delta=139.0,137.0,134.4,129.0,127.3,124.9,73.4,67.1$, $63.5,33.2,30.3,26.5,23.0,18.9,16.2,-4.8 ; \operatorname{LRESIMS}(\mathrm{m} / \mathrm{e}): 387.2\left([\mathrm{M}+\mathrm{Na}]^{+}\right)$; HRESIMS (m/e): calcd. for $\mathrm{C}_{21} \mathrm{H}_{36} \mathrm{O}_{3} \mathrm{SiNa}\left([\mathrm{M}+\mathrm{Na}]^{+}\right)$387.2331, found 387.2340.

tert-Butyldimethyl[5-[(tetrahydro-2H-pyran-2-ylidene)methoxy]pentyloxy]silane

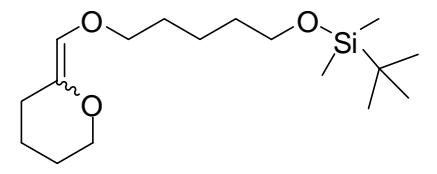

Following the general procedure (GP-1), $\delta$-valerolactone $(45 \mu \mathrm{L}, 0.5 \mathrm{mmol})$ was alkoxymethylenated. The resulting crude mixture was concentrated and chromatographed on basic alumina (activity (II), $\mathrm{CH}_{2} \mathrm{Cl}_{2}$ :hexane $=1: 5$ as eluant) to yield give three fractions: $\mathrm{Z}$ isomer (30.5 mg, 19\% yield), $\mathrm{E}$ isomer $(52.1 \mathrm{mg}, 33 \%$ yield) and a $\mathrm{Z} / \mathrm{E}$ mixture $(30.5 \mathrm{mg}, 19 \%$ yield, $\mathrm{Z}: \mathrm{E}=49: 51)$. The combined yield was $72 \%$ with a stereoselectivity of $\mathrm{Z}: \mathrm{E}=40: 60$.

\section{(Z)-Isomer}

$\mathrm{R}_{f}\left(\right.$ silica- $\left.\mathrm{CH}_{2} \mathrm{Cl}_{2}\right): 0.12 ;{ }^{1} \mathrm{H}$ NMR $\left(400 \mathrm{MHz}, \mathrm{C}_{6} \mathrm{D}_{6}\right): \delta=5.32(\mathrm{~s}, 1 \mathrm{H}), 3.69(\mathrm{~m}$, 2H), $3.56(\mathrm{t}, 2 \mathrm{H}, \mathrm{J}=6.4 \mathrm{~Hz}), 3.48(\mathrm{t}, 2 \mathrm{H}, \mathrm{J}=6.1 \mathrm{~Hz}), 1.87(\mathrm{~m}, 2 \mathrm{H}), 1.58-1.27(\mathrm{~m}, 10 \mathrm{H})$, $0.98(\mathrm{~s}, 9 \mathrm{H}), 0.05(\mathrm{~s}, 6 \mathrm{H}) ;{ }^{13} \mathrm{C}\left[{ }^{1} \mathrm{H}\right] \mathrm{NMR}\left(100 \mathrm{MHz}, \mathrm{C}_{6} \mathrm{D}_{6}\right): \delta=137.4,128.9,72.7,69.8$, 
$63.5,33.3,30.3,27.0,26.6,26.5,24.8,23.0,18.9,-4.8$; LRESIMS $(\mathrm{m} / \mathrm{e}): 337.1$ $\left([\mathrm{M}+\mathrm{Na}]^{+}\right)$; HRESIMS (m/e): calcd. for $\mathrm{C}_{17} \mathrm{H}_{34} \mathrm{O}_{3} \mathrm{SiNa}\left([\mathrm{M}+\mathrm{Na}]^{+}\right)$337.2175, found 337.2172; Anal. calcd. (found) for $\mathrm{C}_{17} \mathrm{H}_{34} \mathrm{O}_{3} \mathrm{Si}$ : C, 64.92 (65.30); $\mathrm{H}, 10.90$ (10.97).

(E)-Isomer

$\mathrm{R}_{f}$ (silica- $\left.\mathrm{CH}_{2} \mathrm{Cl}_{2}\right): 0.19 ;{ }^{1} \mathrm{H}$ NMR (400 MHz, $\left.\mathrm{C}_{6} \mathrm{D}_{6}\right): \delta=6.29(\mathrm{~s}, 1 \mathrm{H}), 3.66(\mathrm{~m}$, 2H), $3.49(\mathrm{t}, 2 \mathrm{H}, \mathrm{J}=6.2 \mathrm{~Hz}), 3.40(\mathrm{t}, 2 \mathrm{H}, \mathrm{J}=6.4 \mathrm{~Hz}), 2.44(\mathrm{t}, 2 \mathrm{H}, \mathrm{J}=6.1 \mathrm{~Hz}), 1.44-1.33$ (m, 10H), 0.99 (s, 9H), $0.06(\mathrm{~s}, 6 \mathrm{H}) ;{ }^{13} \mathrm{C}\left[{ }^{1} \mathrm{H}\right] \mathrm{NMR}\left(100 \mathrm{MHz}, \mathrm{C}_{6} \mathrm{D}_{6}\right): \delta=142.6,133.1$, 72.7, 71.3, 63.5, 32.3, 30.1, 27.0, 26.5, 23.9, 23.0, 18.9, -4.8; LRESIMS (m/e): 337.1 $\left([\mathrm{M}+\mathrm{Na}]^{+}\right)$; HRESIMS (m/e): calcd. for $\mathrm{C}_{17} \mathrm{H}_{34} \mathrm{O}_{3} \mathrm{SiNa}\left([\mathrm{M}+\mathrm{Na}]^{+}\right)$337.2175, found 337.2170.

3-[1-(4-Isobutylphenyl)ethyl]-12,12,13,13-tetramethyl-2,5,11-trioxa-12-silatetradec-3-ene

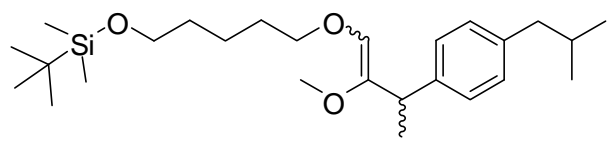

Following the general procedure (GP-1), methyl 2-(4-isobutylphenyl)propanoate $(0.11 \mathrm{~g}, 0.5 \mathrm{mmol})$ was alkoxymethylenated. The resulting crude mixture was concentrated and chromatographed on basic alumina (activity (II), $\mathrm{CH}_{2} \mathrm{Cl}_{2}$ :hexane $=1: 8$ ) to give three fractions: Z-isomer (32 $\mathrm{mg}, 15 \%$ yield), a Z/E mixture 1 (34.1 $\mathrm{mg}, 16 \%$ yield, $Z: E=26: 74)$ and a $Z / E$ mixture $2(68.5 \mathrm{mg}, 32 \%$ yield, $Z: E=65: 35)$. The combined yield was $63 \%$ with a stereoselectivity of $Z: E=63: 37$.

(Z)-Isomer

$\mathrm{R}_{f}$ (silica- $\left.\mathrm{CH}_{2} \mathrm{Cl}_{2}\right): 0.35 ;{ }^{1} \mathrm{H}$ NMR $\left(400 \mathrm{MHz}, \mathrm{C}_{6} \mathrm{D}_{6}\right): \delta=7.34(\mathrm{~d}, 2 \mathrm{H}, \mathrm{J}=8.0$ S-10 
Hz), $7.07(\mathrm{~d}, 2 \mathrm{H}, \mathrm{J}=8.1 \mathrm{~Hz}), 5.45(\mathrm{~s}, 1 \mathrm{H}), 3.74(\mathrm{~s}, 3 \mathrm{H}), 3.49(\mathrm{t}, 2 \mathrm{H}, \mathrm{J}=6.0 \mathrm{~Hz}), 3.38(\mathrm{t}$, $2 \mathrm{H}, \mathrm{J}=6.3 \mathrm{~Hz}), 3.36-3.33(\mathrm{~m}, 1 \mathrm{H}), 2.38(\mathrm{~d}, 2 \mathrm{H}, \mathrm{J}=7.2 \mathrm{~Hz}), 1.77-1.72(\mathrm{~m}, 1 \mathrm{H}), 1.45(\mathrm{~d}$, $3 \mathrm{H}, \mathrm{J}=7.1 \mathrm{~Hz}), 1.48-1.32(\mathrm{~m}, 6 \mathrm{H}), 0.98(\mathrm{~s}, 9 \mathrm{H}), 0.86(\mathrm{~d}, 6 \mathrm{H}, \mathrm{J}=6.6 \mathrm{~Hz}), 0.05(\mathrm{~s}, 6 \mathrm{H})$; ${ }^{13} \mathrm{C}\left[{ }^{1} \mathrm{H}\right] \mathrm{NMR}\left(75 \mathrm{MHz}, \mathrm{C}_{6} \mathrm{D}_{6}\right): \delta=144.6,142.8,140.0,129.7,129.6,128.1,73.0,63.4$, $59.8,45.8,42.4,33.2,30.9,30.2,26.5,23.1,22.9,19.9,18.9,-4.8$; LRESIMS (m/e): $457.3\left([\mathrm{M}+\mathrm{Na}]^{+}\right)$; HRESIMS (m/e): calcd. for $\mathrm{C}_{26} \mathrm{H}_{46} \mathrm{O}_{3} \mathrm{SiNa}\left([\mathrm{M}+\mathrm{Na}]^{+}\right)$457.3114, found 457.3116.

(S)-3-[1-(6-methoxynaphthalen-2-yl)ethyl]-12,12,13,13-tetramethyl-2,5,11-trioxa-12silatetradec-3-ene

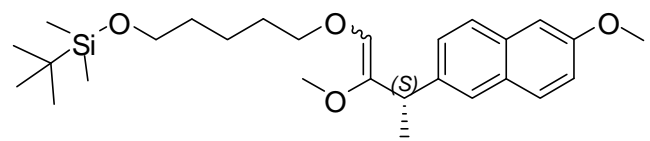

Following the general procedure (GP-1), (S)-Methyl 2-(6-methoxynaphthalen-2yl)propanoate $(0.12 \mathrm{~g}, 0.5 \mathrm{mmol})$ was alkoxymethylenated. The resulting crude mixture was concentrated and chromatographed on basic alumina (activity (II), $\mathrm{CH}_{2} \mathrm{Cl}_{2}$ :hexane = 1:8 as eluant) to give three fractions: Z-isomer (30.8 mg, 13\% yield), E-isomer ( $23 \mathrm{mg}$, $10 \%$ yield) and a $\mathrm{Z} / \mathrm{E}$ mixture $(37 \mathrm{mg}, 16 \%$ yield, $\mathrm{Z}: \mathrm{E}=65: 35)$. The combined yield was $40 \%$ with a stereoselectivity of $Z: E=61: 39$.

(Z)-Isomer

$\mathrm{R}_{f}$ (silica- $\left.\mathrm{CH}_{2} \mathrm{Cl}_{2}\right): 0.29 ;{ }^{1} \mathrm{H}$ NMR $\left(400 \mathrm{MHz}, \mathrm{C}_{6} \mathrm{D}_{6}\right): \delta=7.74(\mathrm{~s}, 1 \mathrm{H}), 7.66(\mathrm{~d}$, $1 \mathrm{H}, \mathrm{J}=8.5 \mathrm{~Hz}), 7.57(\mathrm{~d}, 1 \mathrm{H}, \mathrm{J}=8.6 \mathrm{~Hz}), 7.56(\mathrm{~d}, 1 \mathrm{H}, \mathrm{J}=8.9 \mathrm{~Hz}), 7.20(\mathrm{dd}, 1 \mathrm{H}, \mathrm{J}=2.5$ Hz, J = 8.9 Hz), $6.98(\mathrm{~d}, 1 \mathrm{H}, \mathrm{J}=2.4 \mathrm{~Hz}), 5.51(\mathrm{~s}, 1 \mathrm{H}), 3.75(\mathrm{~s}, 3 \mathrm{H}), 3.49(\mathrm{t}, 2 \mathrm{H}, \mathrm{J}=6.0$ Hz), 3.53-3.47(m, 1H), $3.41(\mathrm{~s}, 3 \mathrm{H}), 3.40(\mathrm{t}, 2 \mathrm{H}, \mathrm{J}=6.2 \mathrm{~Hz}), 1.54(\mathrm{~d}, 3 \mathrm{H}, \mathrm{J}=7.1 \mathrm{~Hz})$, 
1.53-1.35 (m, 6H), $0.98(\mathrm{~s}, 9 \mathrm{H}), 0.05(\mathrm{~m}, 6 \mathrm{H}) ;{ }^{13} \mathrm{C}\left[{ }^{1} \mathrm{H}\right] \mathrm{NMR}\left(100 \mathrm{M} \mathrm{Hz}, \mathrm{C}_{6} \mathrm{D}_{6}\right): \delta=$ 158.3, 144.4, 140.4, 134.4, 130.1, 130.0, 128.7, 127.7, 127.3, 126.5, 119.4, 106.3, 73.0, 63.4, 59.8, 55.1, 42.7, 33.2, 30.2, 26.5, 23.0, 19.7, 18.8, -4.8; LRESIMS (m/e): 481.1 ([M+Na $\left.]^{+}\right)$; Anal. calcd. (found) for $\mathrm{C}_{27} \mathrm{H}_{42} \mathrm{O}_{4} \mathrm{Si}$ : C, 70.70 (70.99); H, 9.23 (9.17).

(E)-Isomer

$\mathrm{R}_{f}\left(\right.$ silica- $\left.\mathrm{CH}_{2} \mathrm{Cl}_{2}\right): 0.48 ;{ }^{1} \mathrm{H}-\mathrm{NMR}\left(400 \mathrm{MHz}, \mathrm{C}_{6} \mathrm{D}_{6}\right): \delta=7.90(\mathrm{~s}, 1 \mathrm{H}), 7.79-7.76$ (m, 1H), $7.68(\mathrm{~d}, 1 \mathrm{H}, \mathrm{J}=8.5 \mathrm{~Hz}), 7.59(\mathrm{~d}, 1 \mathrm{H}, \mathrm{J}=8.9 \mathrm{~Hz}), 7.20-7.17(\mathrm{~m}, 1 \mathrm{H}), 6.95(\mathrm{~d}, 1 \mathrm{H}$, $\mathrm{J}=2.3 \mathrm{~Hz}), 5.60(\mathrm{~s}, 1 \mathrm{H}), 4.71-4.68(\mathrm{q}, 1 \mathrm{H}, \mathrm{J}=7.2 \mathrm{~Hz}), 3.53(\mathrm{t}, 2 \mathrm{H}, \mathrm{J}=5.9 \mathrm{~Hz}), 3.49(\mathrm{t}$, 2H, J = 6.4 Hz), $3.38(\mathrm{~s}, 3 \mathrm{H}), 3.13(\mathrm{~s}, 3 \mathrm{H}), 1.71(\mathrm{~d}, 3 \mathrm{H}, \mathrm{J}=7.3 \mathrm{~Hz}), 1.62-1.57(\mathrm{~m}, 2 \mathrm{H})$, 1.52-1.46 (m, 4H), 0.99 (s, 9H), $0.07(\mathrm{~s}, 6 \mathrm{H}) ;{ }^{13} \mathrm{C}\left[{ }^{1} \mathrm{H}\right] \mathrm{NMR}\left(100 \mathrm{MHz}, \mathrm{C}_{6} \mathrm{D}_{6}\right): \delta=158.1$, $151.1,140.7,134.3,130.1,130.0,128.0,127.2,126.3,124.9,119.3,106.3,73.3,63.5$, $55.5,55.1,38.0,33.3,30.2,26.5,23.2,18.8,18.4,-4.7$; LRESIMS $(\mathrm{m} / \mathrm{e}): 481.2$ $\left([\mathrm{M}+\mathrm{Na}]^{+}\right)$; HRESIMS (m/e): calcd. for $\mathrm{C}_{27} \mathrm{H}_{42} \mathrm{O}_{4} \mathrm{NaSi}\left([\mathrm{M}+\mathrm{Na}]^{+}\right)$481.2750, found 481.2746.

$\underline{\mathrm{N}-[2-[5-(\text { tert-Butyldimethylsilyloxy)pentyloxy]-1-phenylvinyl]-N-methylaniline }}$

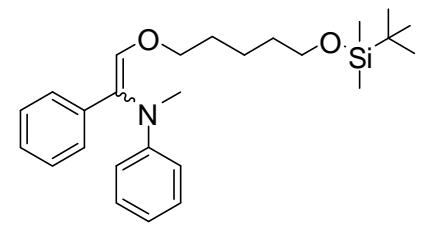

Following the general procedure (GP-1), N-methylbenzanilide (50 mg, 0.24 mmol) was alkoxymethylenated. The resulting crude mixture was concentrated and chromatographed on basic alumina (activity (II), hexane) to give a diastereomeric mixture (52 mg, 51\% yield, $\mathrm{Z}: \mathrm{E}=83: 17)$. 
(Z)-Isomer (not isolated, data were obtained from the above mixture sample)

${ }^{1} \mathrm{H}$ NMR $\left(400 \mathrm{MHz}, \mathrm{C}_{6} \mathrm{D}_{6}\right): \delta=7.30(\mathrm{~d}, 2 \mathrm{H}, \mathrm{J}=7.4 \mathrm{~Hz}), 7.22-7.17(\mathrm{~m}, 2 \mathrm{H})$, 7.15-7.11 (m, 2H), 7.07-7.03 (m, 1H), $6.83(\mathrm{~d}, 2 \mathrm{H}, \mathrm{J}=8.0 \mathrm{~Hz}), 6.78-6.75(\mathrm{~m}, 1 \mathrm{H}), 6.29$ (s, 1H), $3.44(\mathrm{t}, 2 \mathrm{H}, \mathrm{J}=6.3 \mathrm{~Hz}), 3.40(\mathrm{t}, 2 \mathrm{H}, \mathrm{J}=6.4 \mathrm{~Hz}), 3.04(\mathrm{~s}, 3 \mathrm{H}), 1.36-1.27(\mathrm{~m}, 4 \mathrm{H})$, 1.21-1.15 (m, 2H), $0.99(\mathrm{~s}, 9 \mathrm{H}), 0.07(\mathrm{~s}, 6 \mathrm{H}) ;{ }^{13} \mathrm{C}\left[{ }^{1} \mathrm{H}\right] \mathrm{NMR}\left(100 \mathrm{M} \mathrm{Hz}, \mathrm{C}_{6} \mathrm{D}_{6}\right): \delta=149.2$, $143.2,137.9,129.5,129.3,127.2,126.4,125.5,117.5,113.7,73.5,63.4,38.2,33.1,30.3$, 26.5, 22.7, 18.9, -4.8; LRESIMS (m/e): $426.3\left([\mathrm{M}+\mathrm{H}]^{+}\right)$; HRESIMS (m/e): calcd. for $\mathrm{C}_{26} \mathrm{H}_{40} \mathrm{NO}_{2} \mathrm{Si}\left([\mathrm{M}+\mathrm{H}]^{+}\right)$426.2828, found 426.2824.

\section{General Procedure for desilylation (GP-2)}

A THF suspension $(15 \mathrm{~mL})$ of silyl-protected alcohol $(0.45 \mathrm{mmol})$, tetrabutylammonium fluoride trihydrate $(0.28 \mathrm{~g}, 0.9 \mathrm{mmol})$ and $4 \AA$ molecular sieves (5 $\mathrm{mg}$ ) was stirred for $20 \mathrm{~min}$ before solvent evaporation in vacuo. The residue was purified by basic alumina (activity (III)) chromatography.

\section{General Procedure for Esterification (GP-3)}

A dry $\mathrm{CH}_{2} \mathrm{Cl}_{2}$ solution (5 mL) of porphyrinoid acid (5-(4-carboxyphenyl)10,15,20-triphenylporphyrin (TPPA) or ring B-BPD- $7^{2}$-monoacid (BPDA), $0.2 \mathrm{mmol}$ ), alcohol (0.2 mmol), coupling agent 1-ethyl-3-(3-dimethylaminopropyl)carbodiimide (EDC, $0.3 \mathrm{mmol}$ ), and DMAP (25 $\mathrm{mg}, 0.2 \mathrm{mmol}$ ) was stirred for 2 days, protected from the light. The crude mixture was concentrated and purified by basic alumina (activity (II or III)) chromatography or prep. TLC. The entire procedure was protected from the light 
by covering all the reaction and purification apparatus and vessels using aluminum foil. It was found preferable to carry out the work-up and chromatography in darkness during the night.

\section{TPP-ethyl butyrate complex 2}

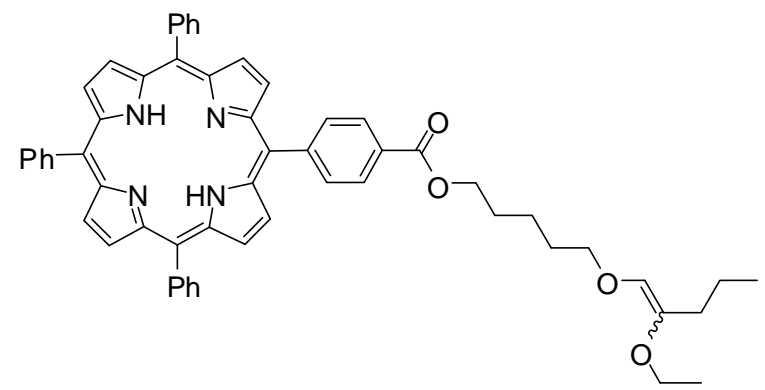

Following the general procedure (GP-2), desilylation of a Z/E mixture of 2,2,3,3tetramethyl-12-propyl-4,10,13-trioxa-3-silapentadec-11-ene $\quad(40 \mathrm{mg}, \quad 0.12 \mathrm{mmol})$, followed by basic alumina chromatography (activity (II), ethyl acetate:hexane $=1: 6$ ) gave 5-(2-ethoxypent-1-enyloxy)pentan-1-ol (15 mg, 58\% yield).

The above alcohol was employed in the esterification of TPPA $(51 \mathrm{mg}, 0.077$ mmol) following the general procedure (GP-3). The crude mixture was concentrated and purified by basic alumina chromatography (activity (II), $\mathrm{CH}_{2} \mathrm{Cl}_{2}$ :hexane $=1: 2$ ) to give $\mathbf{2 Z}$ (16 mg), 2E (27 mg) and a Z/E mixture ( $8 \mathrm{mg})$. The combined yield was $85 \%$.

\section{$2 Z$}

$\mathrm{R}_{f}\left(\right.$ silica- $\left.\mathrm{CH}_{2} \mathrm{Cl}_{2}\right)$ 0.19; ${ }^{1} \mathrm{H}-\mathrm{NMR}\left(400 \mathrm{MHz}, \mathrm{C}_{6} \mathrm{D}_{6}\right): \delta=8.96(\mathrm{~d}, 2 \mathrm{H}, \mathrm{J}=4.8$ Hz), 8.93 (s, 4H), $8.83(\mathrm{~d}, 2 \mathrm{H}, \mathrm{J}=4.8 \mathrm{~Hz}), 8.49$ (d, 2H, J = 8.2 Hz), 8.14-8.09 (m, 6H), $8.08(\mathrm{~d}, 2 \mathrm{H}, \mathrm{J}=8.2 \mathrm{~Hz}), 7.51-7.41(\mathrm{~m}, 9 \mathrm{H}), 5.32(\mathrm{~s}, 1 \mathrm{H}), 4.35(\mathrm{t}, 2 \mathrm{H}, \mathrm{J}=6.6 \mathrm{~Hz}), 4.19(\mathrm{q}$, $2 \mathrm{H}, \mathrm{J}=7.0 \mathrm{~Hz}), 3.42(\mathrm{t}, 2 \mathrm{H}, \mathrm{J}=6.1 \mathrm{~Hz}), 1.94(\mathrm{t}, 2 \mathrm{H}, \mathrm{J}=7.2 \mathrm{~Hz}), 1.66-1.57(\mathrm{~m}, 4 \mathrm{H})$, 
1.52-1.45 (m, 2H), 1.44-1.39 (m, 2H), $1.27(\mathrm{t}, 3 \mathrm{H}, \mathrm{J}=7.0 \mathrm{~Hz}), 0.93(\mathrm{t}, 3 \mathrm{H}, \mathrm{J}=7.4 \mathrm{~Hz})$, $2.13(\mathrm{~s}, 2 \mathrm{H}) ;{ }^{13} \mathrm{C}\left[{ }^{1} \mathrm{H}\right]$ NMR $\left(100 \mathrm{MHz}, \mathrm{C}_{6} \mathrm{D}_{6}\right): \delta=166.9,147.8,143.1,139.3,135.3$, $135.2,132.0,130.8,128.5,128.4,128.2,127.3,121.4,121.2,119.6,72.6,66.4,65.5$, 34.1, 30.0, 29.3, 23.3, 21.4, 16.5, 14.1; LRESIMS (m/e): $857.4\left([\mathrm{M}+\mathrm{H}]^{+}\right)$; HRESIMS (m/e): calcd. for $\mathrm{C}_{57} \mathrm{H}_{53} \mathrm{~N}_{4} \mathrm{O}_{4}\left([\mathrm{M}+\mathrm{H}]^{+}\right)$857.4067, found 857.4069; UV-Vis $\left(\mathrm{CH}_{2} \mathrm{Cl}_{2}\right)$ $\lambda_{\max } / \mathrm{nm}(\log \varepsilon): 418$ (5.75), 516 (4.24), 550 (3.92), 590 (3.79), 645 (3.69).

2E

$\mathrm{R}_{f}\left(\right.$ silica- $\left.\mathrm{CH}_{2} \mathrm{Cl}_{2}\right)$ 0.31; ${ }^{1} \mathrm{H}-\mathrm{NMR}\left(400 \mathrm{MHz}, \mathrm{C}_{6} \mathrm{D}_{6}\right): \delta=8.95(\mathrm{~d}, 2 \mathrm{H}, \mathrm{J}=4.8$ Hz), $8.92(\mathrm{~s}, 4 \mathrm{H}), 8.82(\mathrm{~d}, 2 \mathrm{H}, \mathrm{J}=4.8 \mathrm{~Hz}), 8.50(\mathrm{~d}, 2 \mathrm{H}, \mathrm{J}=8.2 \mathrm{~Hz}), 8.13-8.10(\mathrm{~m}, 6 \mathrm{H})$, $8.07(\mathrm{~d}, 2 \mathrm{H}, \mathrm{J}=8.2 \mathrm{~Hz}), 7.51-7.45(\mathrm{~m}, 9 \mathrm{H}), 5.76(\mathrm{~s}, 1 \mathrm{H}), 4.39(\mathrm{t}, 2 \mathrm{H}, \mathrm{J}=6.6 \mathrm{~Hz}), 3.49(\mathrm{t}$, $2 \mathrm{H}, \mathrm{J}=6.2 \mathrm{~Hz}), 3.42(\mathrm{q}, 2 \mathrm{H}, \mathrm{J}=7.0 \mathrm{~Hz}), 2.55(\mathrm{t}, 2 \mathrm{H}, \mathrm{J}=7.4 \mathrm{~Hz}), 1.81-1.73(\mathrm{~m}, 2 \mathrm{H})$, 1.71-1.64 (m, 2H), 1.62-1.55 (m, 2H), 1.53-1.45 (m, 2H), $1.12(\mathrm{t}, 3 \mathrm{H}, \mathrm{J}=6.9 \mathrm{~Hz}), 1.04(\mathrm{t}$, $3 \mathrm{H}, \mathrm{J}=7.4 \mathrm{~Hz}),-2.15(\mathrm{~s}, 2 \mathrm{H}) ;{ }^{13} \mathrm{C}\left[{ }^{1} \mathrm{H}\right] \mathrm{NMR}\left(100 \mathrm{MHz}, \mathrm{C}_{6} \mathrm{D}_{6}\right): \delta=166.9,147.8,147.7$, $143.1,135.2,130.8,128.7,128.3,127.3,126.6,121.4,121.2,119.6,72.9,65.5,63.3$, 30.9, 30.0, 29.4, 23.4, 21.3, 15.5, 14.5; ESIMS (m/e): $857.4\left([\mathrm{M}+\mathrm{H}]^{+}\right)$; HRESIMS (m/e): calcd. for $\mathrm{C}_{57} \mathrm{H}_{53} \mathrm{~N}_{4} \mathrm{O}_{4}\left([\mathrm{M}+\mathrm{H}]^{+}\right)$857.4067, found 857.4069; UV-Vis $\left(\mathrm{CH}_{2} \mathrm{Cl}_{2}\right)$ $\lambda_{\max } / \mathrm{nm}(\log \varepsilon): 418$ (5.78), 514 (4.32), 549 (4.01), 590 (3.88), 645 (3.79).

\section{TPP-ethyl benzoate complex $3 Z$}




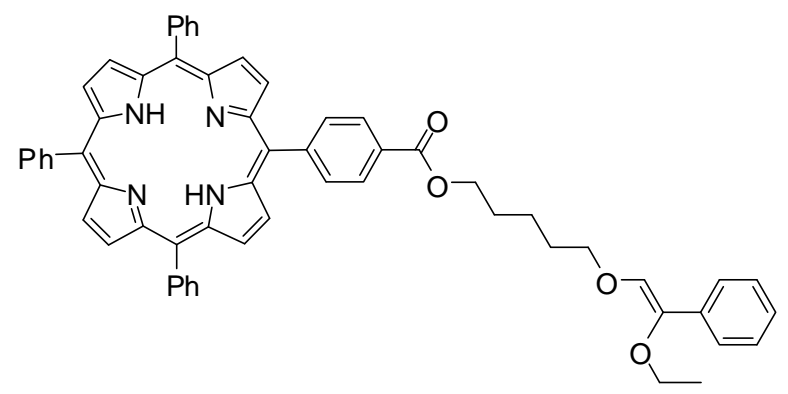

Following the general procedure (GP-2), desilylation of (Z)-2,2,3,3-tetramethyl 12-phenyl-4,10,13-trioxa-3-silapentadec-11-ene $(16.4 \mathrm{mg}, 0.045 \mathrm{mmol})$, followed by basic alumina chromatography (activity (II), ethyl acetate:hexane $=1: 5$ as eluant) gave (Z)-5-(2-ethoxy-2-phenylvinyloxy)pentan-1-ol (7.8 mg, 70\% yield).

The above alcohol was employed in the esterification of TPPA (21 mg, 0.032 mmol) using EDC (10 mg, $0.05 \mathrm{mmol})$ as coupler, following the general procedure (GP3). The crude mixture was purified by basic alumina chromatography (activity (II), $\mathrm{CH}_{2} \mathrm{Cl}_{2}$ :hexane $=1: 1$ as eluant) to give $\mathbf{3 Z}(27 \mathrm{mg}$, quantitative yield $)$.

$$
\mathrm{R}_{f}\left(\text { silica- } \mathrm{CH}_{2} \mathrm{Cl}_{2}\right) \text { 0.22; }{ }^{1} \mathrm{H}-\mathrm{NMR}\left(400 \mathrm{MHz}, \mathrm{C}_{6} \mathrm{D}_{6}\right): \delta=8.95(\mathrm{~d}, 2 \mathrm{H}, \mathrm{J}=4.8
$$

Hz), $8.92(\mathrm{~s}, 4 \mathrm{H}), 8.82(\mathrm{~d}, 2 \mathrm{H}, \mathrm{J}=4.7 \mathrm{~Hz}), 8.49$ (d, 2H, J = 8.1 Hz), 8.13-8.09 (m, 6H), $8.08(\mathrm{~d}, 2 \mathrm{H}, \mathrm{J}=8.1 \mathrm{~Hz}), 7.53(\mathrm{~d}, 2 \mathrm{H}, \mathrm{J}=7.3 \mathrm{~Hz}), 7.49-7.43(\mathrm{~m}, 9 \mathrm{H}), 7.17-7.13(\mathrm{~m}, 2 \mathrm{H})$, 7.07-7.03 (m, 1H), $6.16(\mathrm{~s}, 1 \mathrm{H}), 4.35(\mathrm{t}, 2 \mathrm{H}, \mathrm{J}=6.5 \mathrm{~Hz}), 4.05(\mathrm{q}, 2 \mathrm{H}, \mathrm{J}=7.0 \mathrm{~Hz}), 3.49(\mathrm{t}$, $2 \mathrm{H}, \mathrm{J}=6.1 \mathrm{~Hz}), 1.65-1.59(\mathrm{~m}, 2 \mathrm{H}), 1.50-1.39(\mathrm{~m}, 4 \mathrm{H}), 1.30(\mathrm{t}, 3 \mathrm{H}, \mathrm{J}=7.0 \mathrm{~Hz}),-2.14(\mathrm{~s}$, $2 \mathrm{H}) ;{ }^{13} \mathrm{C}\left[{ }^{1} \mathrm{H}\right] \mathrm{NMR}\left(100 \mathrm{MHz}, \mathrm{C}_{6} \mathrm{D}_{6}\right): \delta=166.8,147.8,143.1,136.9,135.2,134.2$, 130.7, 129.0, 128.6, 128.2, 127.3, 125.0, 121.4, 121.2, 119.5, 73.1, 67.2, 65.4, 30.0, 29.2, 23.1, 16.2; LRESIMS (m/e): $891.4\left([\mathrm{M}+\mathrm{H}]^{+}\right)$; $\operatorname{HRESIMS~(m/e):~calcd.~for~} \mathrm{C}_{60} \mathrm{H}_{51} \mathrm{~N}_{4} \mathrm{O}_{4}$ $\left([\mathrm{M}+\mathrm{H}]^{+}\right)$891.3910, found 891.3909; UV-Vis $\left(\mathrm{CH}_{2} \mathrm{Cl}_{2}\right) \lambda_{\max } / \mathrm{nm}(\log \varepsilon): 418$ (5.58), 515 (4.12), 550 (3.81), 589 (3.68), 645 (3.58). 


\section{$\underline{\text { TPP- } \delta \text {-valerolactone complex } 4}$}

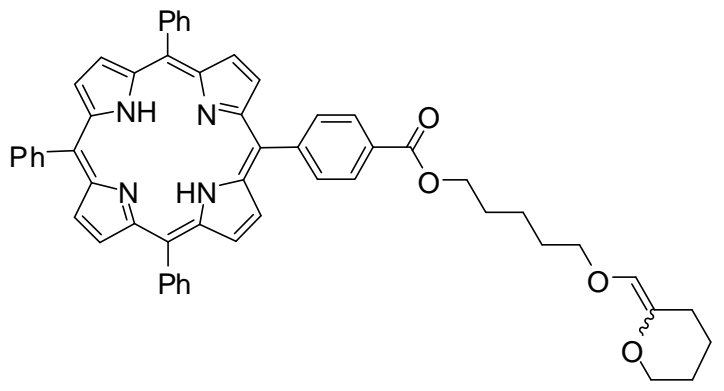

Following the general procedure (GP-2), desilylation of tert-butyldimethyl[5 [(tetrahydro-2H-pyran-2-ylidene)methoxy]pentyloxy]silane $(30.5 \mathrm{mg}, 0.095 \mathrm{mmol})$, followed by basic alumina chromatography (activity (II), ethyl acetate:hexane $=1: 4$ as eluant) gave 5-[(tetrahydro-2H-pyran-2-ylidene)methoxy]pentan-1-ol (14 mg, 71\% yield).

The above alcohol was employed in the esterification of TPPA (46 mg, 0.07 mmol) using EDC (19 mg, $0.1 \mathrm{mmol}$ ) as coupler, following the general procedure (GP-3). The crude mixture was concentrated and purified by basic alumina chromatography (activity (II), $\mathrm{CH}_{2} \mathrm{Cl}_{2}$ :hexane $=1: 1$ as eluant) to give $\mathbf{4 Z}(22.5 \mathrm{mg}), \mathbf{4 E}(25 \mathrm{mg})$ and a $\mathrm{Z} / \mathrm{E}$ mixture (4.5 mg). The combined yield was $88 \%$.

\section{$4 \mathrm{Z}$}

$\mathrm{R}_{f}\left(\right.$ silica- $\left.\mathrm{CH}_{2} \mathrm{Cl}_{2}\right)$ 0.09; ${ }^{1} \mathrm{H}-\mathrm{NMR}\left(400 \mathrm{MHz}, \mathrm{d}_{6}\right.$-acetone): $\delta=8.86-8.85(\mathrm{~m}$, 8H), $8.45(\mathrm{~d}, 2 \mathrm{H}, \mathrm{J}=8.0 \mathrm{~Hz}), 8.36(\mathrm{~d}, 2 \mathrm{H}, \mathrm{J}=8.1 \mathrm{~Hz}), 8.25-8.22(\mathrm{~m}, 6 \mathrm{H}), 7.84-7.78(\mathrm{~m}$, 9H), $5.47(\mathrm{~s}, 1 \mathrm{H}), 4.50(\mathrm{t}, 2 \mathrm{H}, \mathrm{J}=6.5 \mathrm{~Hz}), 3.77-3.70(\mathrm{~m}, 4 \mathrm{H}), 2.07-2.01(\mathrm{~m}, 2 \mathrm{H}), 1.96-$ $1.90(\mathrm{~m}, 4 \mathrm{H}), 1.76-1.64(\mathrm{~m}, 4 \mathrm{H}), 1.61-1.59(\mathrm{~m}, 2 \mathrm{H}),-2.75(\mathrm{~s}, 2 \mathrm{H}) ;{ }^{13} \mathrm{C}\left[{ }^{1} \mathrm{H}\right] \mathrm{NMR}(100$ $\mathrm{MHz}, \mathrm{d}_{6}$-acetone): $\delta=167.0,147.6,142.8,135.5,135.3,131.1,129.0,128.8,127.8$, 121.6, 121.4, 119.8, 72.5, 69.9, 65.9, 30.2, 29.5, 26.9, 26.7, 24.9, 23.4; LRESIMS (m/e): 
$841.3\left([\mathrm{M}+\mathrm{H}]^{+}\right)$; HRESIMS (m/e): calcd. for $\mathrm{C}_{56} \mathrm{H}_{49} \mathrm{~N}_{4} \mathrm{O}_{4}\left([\mathrm{M}+\mathrm{H}]^{+}\right)$841.3754, found 841.3752; UV-Vis $\left(\mathrm{CH}_{2} \mathrm{Cl}_{2}\right) \lambda_{\max } / \mathrm{nm}(\log \varepsilon): 418$ (5.73), 514 (4.22), 550 (3.88), 590 (3.74), 645 (3.64).

TPP-ibuprofen (methyl ester) complex $\mathbf{5}$

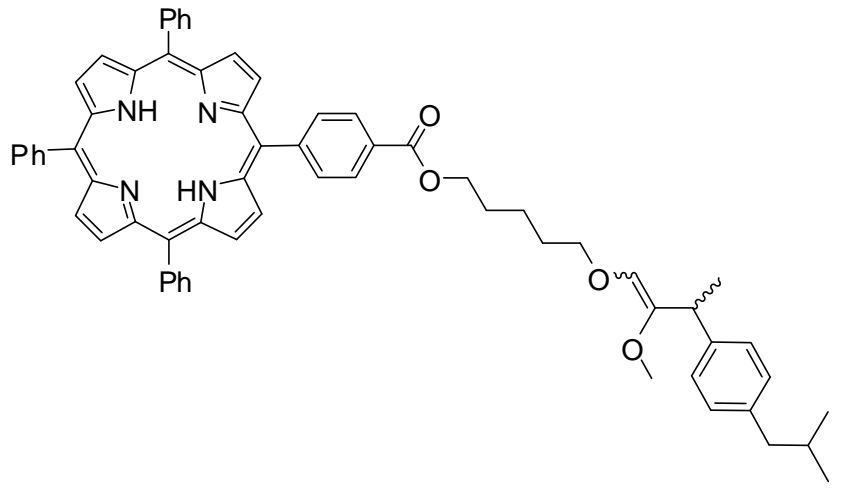

Following the general procedure (GP-2), desilylation of a Z/E mixture of 3-[1(4-isobutylphenyl)ethyl]-12,12,13,13-tetramethyl-2,5,11-trioxa-12-silatetradec-3-ene (55 $\mathrm{mg}, 0.127 \mathrm{mmol}$ ), followed by basic alumina chromatography (activity (II), ethyl acetate:hexane = 1:4) gave 5-[3-(4-isobutylphenyl)-2-methoxybut-1-enyloxy]pentan-1-ol (30 mg, 75\% yield).

Half of the above alcohol (the other half was employed in the synthesis of 6) was employed in the esterification of TPPA $(36 \mathrm{mg}, 0.055 \mathrm{mmol})$ following the general procedure (GP-3). The crude mixture was concentrated and purified by silica prep. TLC $\left(\mathrm{CH}_{2} \mathrm{Cl}_{2}\right)$ to give $5 \mathrm{Z}(31 \mathrm{mg})$ and $5 \mathrm{E}(14 \mathrm{mg})$. The combined yield was $98 \%$.

$5 Z$

$\mathrm{R}_{f}\left(\right.$ silica- $\left.\mathrm{CH}_{2} \mathrm{Cl}_{2}\right)$ 0.36; ${ }^{1} \mathrm{H}-\mathrm{NMR}\left(400 \mathrm{MHz}, \mathrm{C}_{6} \mathrm{D}_{6}\right): \delta=8.96(\mathrm{~d}, 2 \mathrm{H}, \mathrm{J}=4.8$ Hz), $8.93(\mathrm{~s}, 4 \mathrm{H}), 8.82(\mathrm{~d}, 2 \mathrm{H}, \mathrm{J}=4.8 \mathrm{~Hz}), 8.48(\mathrm{~d}, 2 \mathrm{H}, \mathrm{J}=8.1 \mathrm{~Hz}), 8.13-8.09(\mathrm{~m}, 6 \mathrm{H})$, 
$8.07(\mathrm{~d}, 2 \mathrm{H}, \mathrm{J}=8.1 \mathrm{~Hz}), 7.49-7.43(\mathrm{~m}, 9 \mathrm{H}), 7.35(\mathrm{~d}, 2 \mathrm{H}, \mathrm{J}=8.0 \mathrm{~Hz}), 7.05(\mathrm{~d}, 2 \mathrm{H}, \mathrm{J}=8.0$ Hz), $5.47(\mathrm{~s}, 1 \mathrm{H}), 4.34(\mathrm{t}, 2 \mathrm{H}, \mathrm{J}=6.5 \mathrm{~Hz}), 3.77(\mathrm{~s}, 3 \mathrm{H}), 3.39-3.34(\mathrm{~m}, 3 \mathrm{H}), 2.32(\mathrm{~d}, 2 \mathrm{H}, \mathrm{J}$ $=7.2 \mathrm{~Hz}), 1.76-1.67(\mathrm{~m}, 1 \mathrm{H}), 1.63-1.56(\mathrm{~m}, 2 \mathrm{H}), 1.46(\mathrm{~d}, 3 \mathrm{H}, \mathrm{J}=7.1 \mathrm{~Hz}), 1.43-1.32(\mathrm{~m}$, $4 \mathrm{H}), 0.80(\mathrm{~d}, 6 \mathrm{H}, \mathrm{J}=6.6 \mathrm{~Hz}),-2.14(\mathrm{~s}, 2 \mathrm{H}) ;{ }^{13} \mathrm{C}\left[{ }^{1} \mathrm{H}\right] \mathrm{NMR}\left(75 \mathrm{MHz}, \mathrm{C}_{6} \mathrm{D}_{6}\right): \delta=166.9$, $147.8,144.7,143.1,142.8,140.0,135.3,130.8,129.7,129.6,128.6,128.4,128.3,127.4$, $121.4,121.3,119.672 .7,65.4,59.9,45.7,42.4,30.8,30.0,29.2,23.2,22.8,19.8$; LRESIMS (m/e): $983.7\left([\mathrm{M}+\mathrm{Na}]^{+}\right)$; HRESIMS (m/e): calcd. for $\mathrm{C}_{65} \mathrm{H}_{60} \mathrm{~N}_{4} \mathrm{O}_{4} \mathrm{Na}$ $\left([\mathrm{M}+\mathrm{Na}]^{+}\right)$983.4512, found 983.4515; UV-Vis $\left(\mathrm{CH}_{2} \mathrm{Cl}_{2}\right) \lambda_{\max } / \mathrm{nm}(\log \varepsilon): 418$ (5.60), 515 (4.14), 549 (3.84), 590 (3.71), 645 (3.62).

\section{BPD-ibuprofen (methyl ester) complex 6}

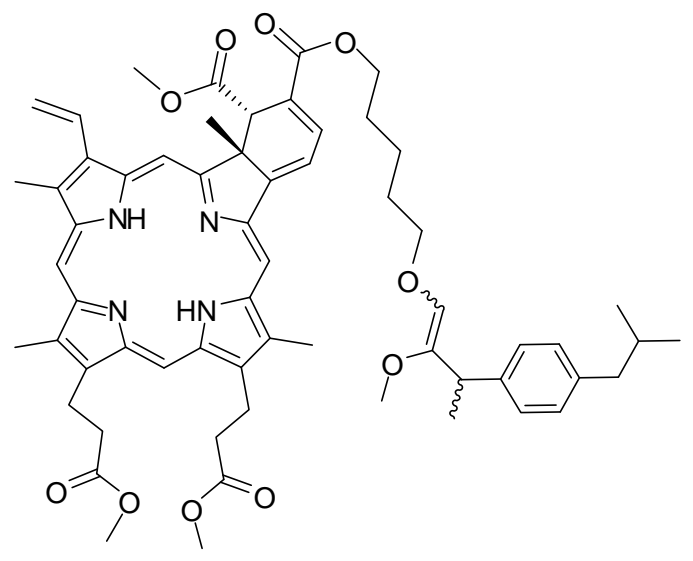

The second half of the alcohol 5-[3-(4-isobutylphenyl)-2-methoxybut-1-enyloxy] pentan-1-ol generated from the synthesis of 5 was used in the esterification of BPDA (39 $\mathrm{mg}, 0.055 \mathrm{mmol}$ ) following the general procedure (GP-3). The crude mixture was concentrated and purified by silica prep. TLC $\left(1 \% \mathrm{MeOH}\right.$ in $\left.\mathrm{CH}_{2} \mathrm{Cl}_{2}\right)$ to give $\mathbf{6 Z}(18 \mathrm{mg})$ and $6 \mathrm{E}(11 \mathrm{mg})$. The combined yield was $58 \%$. 
6Z (two pairs of diastereomers in a 1:1 ratio )

$\mathrm{R}_{f}$ (silica-1\% MeOH in $\mathrm{CH}_{2} \mathrm{Cl}_{2}$ ) 0.16; ${ }^{1} \mathrm{H}-\mathrm{NMR}$ (400 MHz, $\mathrm{d}_{6}$-acetone, signals of diastereomers are completely coincident): $\delta=9.84(\mathrm{~s}, 1 \mathrm{H}), 9.77(\mathrm{~s}, 1 \mathrm{H}), 9.51(\mathrm{~s}, 1 \mathrm{H})$, $9.40(\mathrm{~s}, 1 \mathrm{H}), 8.28(\mathrm{dd}, 1 \mathrm{H}, \mathrm{J}=11.6 \mathrm{~Hz}, \mathrm{~J}=17.9 \mathrm{~Hz}), 7.82(\mathrm{~d}, 1 \mathrm{H}, \mathrm{J}=5.7 \mathrm{~Hz}), 7.71(\mathrm{~d}, 1 \mathrm{H}$, $\mathrm{J}=5.7 \mathrm{~Hz}), 7.19(\mathrm{~d}, 2 \mathrm{H}, \mathrm{J}=8.0 \mathrm{~Hz}), 7.02(\mathrm{~d}, 2 \mathrm{H}, \mathrm{J}=8.0 \mathrm{~Hz}), 6.45(\mathrm{dd}, 1 \mathrm{H}, \mathrm{J}=1.2 \mathrm{~Hz}, \mathrm{~J}$ $=17.9 \mathrm{~Hz}), 6.17(\mathrm{dd}, 1 \mathrm{H}, \mathrm{J}=1.2 \mathrm{~Hz}, \mathrm{~J}=11.6 \mathrm{~Hz}), 5.70(\mathrm{~s}, 1 \mathrm{H}), 5.18(\mathrm{~s}, 1 \mathrm{H}), 4.43-4.32$ (m, 2H), 4.18-4.09 (m, 2H), $4.10(\mathrm{t}, 2 \mathrm{H}, \mathrm{J}=7.6 \mathrm{~Hz}), 3.77(\mathrm{t}, 2 \mathrm{H}, \mathrm{J}=6.3 \mathrm{~Hz}), 3.65(\mathrm{~s}, 3 \mathrm{H})$, $3.62(\mathrm{~s}, 3 \mathrm{H}), 3.60(\mathrm{~s}, 3 \mathrm{H}), 3.57(\mathrm{~s}, 3 \mathrm{H}), 3.34(\mathrm{~s}, 3 \mathrm{H}), 3.32(\mathrm{~s}, 3 \mathrm{H}), 3.35-3.29(\mathrm{~m}, 1 \mathrm{H})$, 3.16-3.12 (m, 4H), 2.98 (s, 3H), 2.36 (d, 2H, J = 7.2 Hz), 1.89-1.63 (m, 10H), 1.31 (d, 3H, $\mathrm{J}=7.2 \mathrm{~Hz}), 0.80(\mathrm{~d}, 6 \mathrm{H}, \mathrm{J}=6.8 \mathrm{~Hz}),-2.25(\mathrm{~s}, 2 \mathrm{H}) ;{ }^{13} \mathrm{C}\left[{ }^{1} \mathrm{H}\right] \mathrm{NMR}\left(100 \mathrm{MHz}, \mathrm{d}_{6}\right.$-acetone $)$ $\delta=173.9,173.7,171.1,167.5,166.8,156.7,154.1,152.9,152.7,144.4,143.0,141.5$ $140.0,139.5,138.6,137.8,137.6,137.3,135.1,134.7,134.4,132.5,131.8,130.6,130.3$, $129.6,128.1,123.9,121.3,114.6,100.9,99.9,94.6,92.8,73.1,65.5,59.7,53.5,51.8$, $51.6,48.8,45.6,42.1,37.5,37.0,31.0,30.0,29.5,28.1,23.5,22.6,22.3,21.8,19.6,12.5$, 11.6, 11.1; LRESIMS (m/e): $1021.3\left([\mathrm{M}+\mathrm{H}]^{+}\right)$; $\operatorname{HRESIMS~(m/e):~calcd.~for~} \mathrm{C}_{61} \mathrm{H}_{73} \mathrm{~N}_{4} \mathrm{O}_{10}$ $\left([\mathrm{M}+\mathrm{H}]^{+}\right)$1021.5327, found 1021.5326; UV-Vis $\left(\mathrm{CH}_{2} \mathrm{Cl}_{2}\right) \lambda_{\max } / \mathrm{nm}(\log \varepsilon): 432$ (4.54), 581 (3.97), 629 (3.78), 690 (4.21).

\section{$\underline{\text { TPP-naproxen (methyl ester) complex } 7}$}




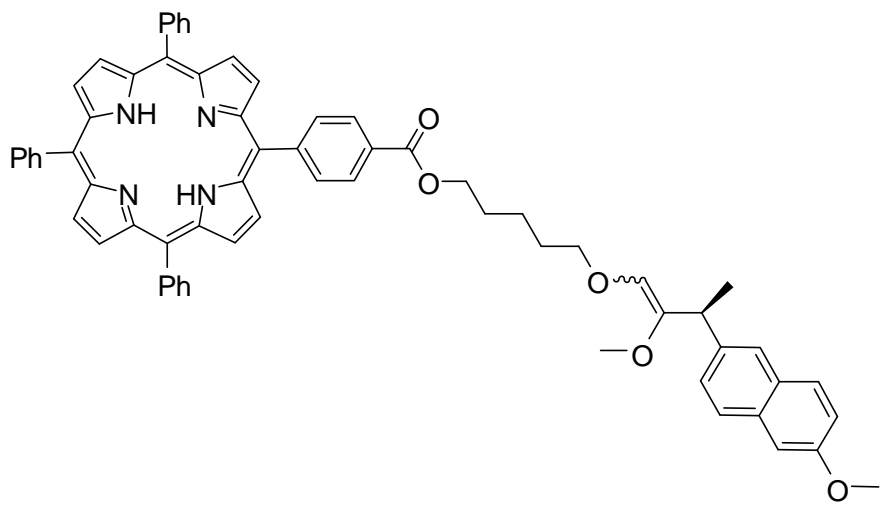

Following the general procedure (GP-2), desilylation of (S)-3-[1-(6methoxynaphthalen-2-yl)ethyl]-12,12,13,13-tetramethyl-2,5,11-trioxa-12-silatetradec-3ene (37 mg, $0.08 \mathrm{mmol}$ ), followed by basic alumina chromatography (activity (II), ethyl acetate:hexane $=1: 4$ as eluant) gave $(S)$-5-[2-methoxy-3-(6-methoxynaphthalen-2-yl)but1 -enyloxy]pentan-1-ol (24 mg, 86\% yield).

The above alcohol was employed in the esterification of TPPA (46 mg, 0.07 mmol) using EDC (23 mg, $0.12 \mathrm{mmol}$ ) as coupler, following the general procedure (GP3). The crude mixture was purified by silica prep. TLC $\left(\mathrm{CH}_{2} \mathrm{Cl}_{2}\right.$ as eluant) to give $\mathbf{7 Z}$ (34 $\mathrm{mg})$ and 7E (21 mg). The combined yield was $80 \%$.

\section{$7 \mathrm{Z}$}

$$
\mathrm{R}_{f}\left(\text { silica- } \mathrm{CH}_{2} \mathrm{Cl}_{2}\right) 0.36 ;{ }^{1} \mathrm{H}-\mathrm{NMR}\left(400 \mathrm{MHz}, \mathrm{C}_{6} \mathrm{D}_{6}\right): \delta=8.95(\mathrm{~d}, 2 \mathrm{H}, \mathrm{J}=4.8
$$

$\mathrm{Hz}), 8.93(\mathrm{~s}, 4 \mathrm{H}), 8.81(\mathrm{~d}, 2 \mathrm{H}, \mathrm{J}=4.8 \mathrm{~Hz}), 8.48(\mathrm{~d}, 2 \mathrm{H}, \mathrm{J}=8.1 \mathrm{~Hz}), 8.13-8.10(\mathrm{~m}, 6 \mathrm{H})$, $8.06(\mathrm{~d}, 2 \mathrm{H}, \mathrm{J}=8.1 \mathrm{~Hz}), 7.73(\mathrm{~s}, 1 \mathrm{H}), 7.64(\mathrm{~d}, 1 \mathrm{H}, \mathrm{J}=8.5 \mathrm{~Hz}), 7.57(\mathrm{dd}, 1 \mathrm{H}, \mathrm{J}=1.6 \mathrm{~Hz}, \mathrm{~J}$ $=8.5 \mathrm{~Hz}), 7.53(\mathrm{~d}, 1 \mathrm{H}, \mathrm{J}=9.0 \mathrm{~Hz}), 7.48-7.42(\mathrm{~m}, 9 \mathrm{H}), 7.16-7.12(\mathrm{~m}, 1 \mathrm{H}), 6.91(\mathrm{~d}, 1 \mathrm{H}, \mathrm{J}$ $=2.3 \mathrm{~Hz}), 5.53(\mathrm{~s}, 1 \mathrm{H}), 4.35(\mathrm{t}, 2 \mathrm{H}, \mathrm{J}=6.5 \mathrm{~Hz}), 3.78(\mathrm{~s}, 3 \mathrm{H}), 3.52(\mathrm{q}, 1 \mathrm{H}, \mathrm{J}=7.1 \mathrm{~Hz})$, $3.40(\mathrm{t}, 2 \mathrm{H}, \mathrm{J}=6.1 \mathrm{~Hz}), 3.31(\mathrm{~s}, 3 \mathrm{H}), 1.63-1.58(\mathrm{~m}, 2 \mathrm{H}), 1.55(\mathrm{~d}, 3 \mathrm{H}, \mathrm{J}=7.1 \mathrm{~Hz}), 1.50-$ 
$1.44(\mathrm{~m}, 2 \mathrm{H}), 1.40-1.32(\mathrm{~m}, 2 \mathrm{H}),-2.14(\mathrm{~s}, 2 \mathrm{H}) ;{ }^{13} \mathrm{C}\left[{ }^{1} \mathrm{H}\right] \mathrm{NMR}\left(100 \mathrm{MHz}, \mathrm{C}_{6} \mathrm{D}_{6}\right): \delta=166$.

$9,158.3,147.8,144.6,143.1,140.3,135.3,134.4,130.7,130.1,130.0,128.7,128.3$,

127.7, 127.4, 127.3, 126.5, 121.4, 121.2, 119.6, 119.5, 106.3, 72.8, 65.4, 59.9, 55.1, 42.7, 30.0, 29.2, 23.2, 19.7; LRESIMS (m/e): $985.5\left([\mathrm{M}+\mathrm{H}]^{+}\right)$; HRESIMS (m/e): calcd. for $\mathrm{C}_{66} \mathrm{H}_{57} \mathrm{~N}_{4} \mathrm{O}_{5}\left([\mathrm{M}+\mathrm{H}]^{+}\right)$985.4329, found 985.4332; UV-Vis $\left(\mathrm{CH}_{2} \mathrm{Cl}_{2}\right) \lambda_{\max } / \mathrm{nm}(\log \varepsilon): 418$ (5.63), 515 (4.13), 550 (3.84), 590 (3.73), 645 (3.64).

BPD-naproxen (methyl ester) complex 8

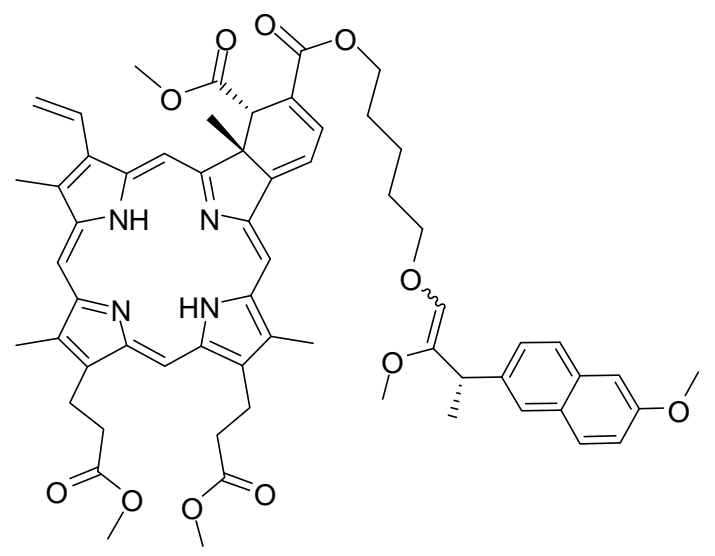

Following the general procedure (GP-2), desilylation of (S)-3-[1-(6methoxynaphthalen-2-yl)ethyl]-12,12,13,13-tetramethyl-2,5,11-trioxa-12-silatetradec-3ene (37 mg, $0.08 \mathrm{mmol}$ ), followed by basic alumina chromatography (activity (II), ethyl acetate:hexane $=1: 4$ as eluant) gave (S)-5-[2-methoxy-3-(6-methoxynaphthalen-2-yl)but1-enyloxy]pentan-1-ol (23 mg, 82\% yield).

The above alcohol was employed in the esterification of BPDA (39 mg, 0.055 mmol) using EDC (23 mg, $0.12 \mathrm{mmol})$ as coupler, following the general procedure (GP3). The crude mixture was purified by silica prep. TLC $\left(\mathrm{CH}_{2} \mathrm{Cl}_{2}\right.$ as eluant) to give $\mathbf{8 Z}$ (19 $\mathrm{mg})$ and $\mathbf{8 E}(14 \mathrm{mg})$. The combined yield was $58 \%$. 
$\mathbf{8 Z}$ (a pair of diastereomers in a 1:1 ratio )

$\mathrm{R}_{f}\left(\right.$ silica- $\left.\mathrm{CH}_{2} \mathrm{Cl}_{2}\right)$ 0.08; ${ }^{1} \mathrm{H}-\mathrm{NMR}\left(300 \mathrm{MHz}, \mathrm{C}_{6} \mathrm{D}_{6}\right.$, signals of the diastereomers are essentially coincident and for each diastereomer, one proton was integrated as $0.5 \mathrm{H}$ ): $\delta=9.95(\mathrm{~s}, 1 \mathrm{H}), 9.73(\mathrm{~s}, 1 \mathrm{H}), 9.47(\mathrm{~s}, 1 \mathrm{H}), 9.17(\mathrm{~s}, 1 \mathrm{H}), 8.09(\mathrm{dd}, 1 \mathrm{H}, \mathrm{J}=11.6 \mathrm{~Hz}, \mathrm{~J}=$ $17.8 \mathrm{~Hz}), 7.98(\mathrm{~d}, 1 \mathrm{H}, \mathrm{J}=5.7 \mathrm{~Hz}), 7.75(\mathrm{~s}, 1 \mathrm{H}), 7.66(\mathrm{~d}, 1 \mathrm{H}, \mathrm{J}=8.4 \mathrm{~Hz}), 7.59(\mathrm{~d}, 1 \mathrm{H}, \mathrm{J}=$ $8.3 \mathrm{~Hz}), 7.56(\mathrm{~d}, 1 \mathrm{H}, \mathrm{J}=8.9 \mathrm{~Hz}), 7.18-7.12(\mathrm{~m}, 2 \mathrm{H}), 6.92(\mathrm{~s}, 1 \mathrm{H}), 6.41(\mathrm{dd}, 1 \mathrm{H}, \mathrm{J}=1.4$ $\mathrm{Hz}, \mathrm{J}=17.8 \mathrm{~Hz}), 6.02(\mathrm{dd}, 1 \mathrm{H}, \mathrm{J}=1.4 \mathrm{~Hz}, \mathrm{~J}=11.6 \mathrm{~Hz}), 5.55(\mathrm{~s}, 1 \mathrm{H}), 5.44(\mathrm{~s}, 1 \mathrm{H}), 4.40-$ $4.20(\mathrm{~m}, 2 \mathrm{H}), 4.21(\mathrm{t}, 2 \mathrm{H}, \mathrm{J}=7.5 \mathrm{~Hz}), 4.13-4.05(\mathrm{~m}, 2 \mathrm{H}), 3.80(\mathrm{~s}, 3 \mathrm{H}), 3.54(\mathrm{q}, 1 \mathrm{H}, \mathrm{J}=$ $7.2 \mathrm{~Hz}), 3.42(\mathrm{t}, 2 \mathrm{H}, \mathrm{J}=6.3 \mathrm{~Hz}), 3.31(\mathrm{~s}, 3 \times 0.5 \mathrm{H}), 3.30(\mathrm{~s}, 3 \times 0.5 \mathrm{H}), 3.29(\mathrm{~s}, 3 \mathrm{H}), 3.28(\mathrm{~s}$, $3 \mathrm{H}), 3.24(\mathrm{~s}, 3 \mathrm{H}), 3.23(\mathrm{~s}, 3 \mathrm{H}), 3.16(\mathrm{t}, 2 \mathrm{H}, \mathrm{J}=7.3 \mathrm{~Hz}), 3.04(\mathrm{t}, 2 \mathrm{H}, \mathrm{J}=7.2 \mathrm{~Hz}), 3.02(\mathrm{~s}$, $3 \mathrm{H}), 2.68(\mathrm{~s}, 3 \mathrm{H}), 1.70(\mathrm{~s}, 3 \mathrm{H}), 1.56(\mathrm{~d}, 3 \mathrm{H}, \mathrm{J}=7.1 \mathrm{~Hz}), 1.65-1.15(\mathrm{~m}, 6 \mathrm{H}),-1.75$ to 1.9(m, 2H); $\operatorname{ESIMS~(m/e):~} 1045.3\left([\mathrm{M}+\mathrm{H}]^{+}\right)$; HRESIMS (m/e): calcd. for $\mathrm{C}_{62} \mathrm{H}_{69} \mathrm{~N}_{4} \mathrm{O}_{11}$ $\left([\mathrm{M}+\mathrm{H}]^{+}\right)$1045.4963, found 1045.4976; UV-Vis $\left(\mathrm{CH}_{2} \mathrm{Cl}_{2}\right) \lambda_{\max } / \mathrm{nm}: 353,430,581,630$, 690

\section{$\underline{\text { TPP- (N-methylbenzanilide) complex } 9}$}

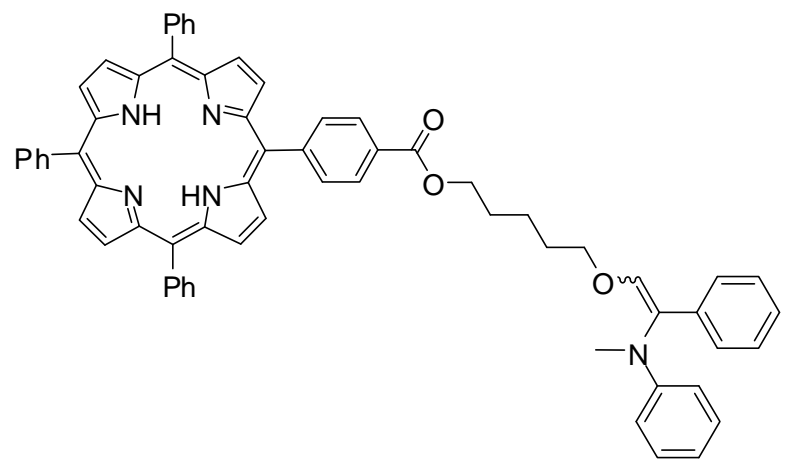

Following the general procedure (GP-2), desilylation of a Z/E mixture of N-[2[5-(tert-butyldimethylsilyloxy)pentyloxy]-1-phenylvinyl]-N-methylaniline (33 mg, 0.076 
mmol), followed by basic alumina chromatography (activity (II), ethyl acetate:hexane $=$ 1:4) gave 5-[2-(methylphenylamino)-2-phenylvinyloxy]pentan-1-ol (15 mg, 64\% yield).

The above alcohol was employed in the esterification of TPPA (33 mg, 0.05 mmol) following the general procedure (GP-3). The crude mixture was concentrated and purified by basic alumina chromatography (activity (II), $\mathrm{CH}_{2} \mathrm{Cl}_{2}$ :hexane $=1: 2$ ) to give $\mathbf{9}$ as a $\mathrm{Z} / \mathrm{E}$ mixture $(37.5 \mathrm{mg}, 81 \%$ yield, $\mathrm{Z}: \mathrm{E}=4: 1)$.

9Z (not isolated, data were obtained from the above mixture)

$$
\mathrm{R}_{f}\left(\text { silica- } \mathrm{CH}_{2} \mathrm{Cl}_{2}\right) \text { 0.65; }{ }^{1} \mathrm{H}-\mathrm{NMR}\left(400 \mathrm{MHz}, \mathrm{C}_{6} \mathrm{D}_{6}\right): \delta=8.96(\mathrm{~d}, 2 \mathrm{H}, \mathrm{J}=4.8
$$

Hz), $8.93(\mathrm{~s}, 4 \mathrm{H}), 8.83$ (d, 2H, J = 4.8 Hz), 8.49 (d, 2H, J = 8.3 Hz), 8.14-8.06 (m, 8H), 7.49-7.41 (m, 9H), $7.33(\mathrm{~d}, 2 \mathrm{H}, \mathrm{J}=7.2 \mathrm{~Hz}), 7.25-7.20(\mathrm{~m}, 2 \mathrm{H}), 7.14-7.08(\mathrm{~m}, 2 \mathrm{H}), 7.06-$ $7.00(\mathrm{~m}, 1 \mathrm{H}), 6.86(\mathrm{~d}, 2 \mathrm{H}, \mathrm{J}=7.9 \mathrm{~Hz}), 6.82-6.76(\mathrm{~m}, 1 \mathrm{H}), 6.30(\mathrm{~s}, 1 \mathrm{H}), 4.30(\mathrm{t}, 2 \mathrm{H}, \mathrm{J}=$ 6.6 Hz), 3.37 (t, 2H, J = 6.1 Hz), $3.05(\mathrm{~s}, 3 \mathrm{H}), 1.54-1.47(\mathrm{~m}, 2 \mathrm{H}), 1.39-1.17(\mathrm{~m}, 4 \mathrm{H})$, -

$2.13(\mathrm{~s}, 2 \mathrm{H}) ;{ }^{13} \mathrm{C}\left[{ }^{1} \mathrm{H}\right] \mathrm{NMR}\left(100 \mathrm{MHz}, \mathrm{C}_{6} \mathrm{D}_{6}\right): \delta=166.9,149.3,147.8,143.1,137.9$, $135.3,130.8,129.6,129.3,128.8,128.3,127.4,127.3,126.7,125.6,121.5,121.3,119.6$, 117.6, 113.8, 73.1, 65.5, 38.2, 30.1, 29.1, 22.9; LRESIMS (m/e): $952.4\left([\mathrm{M}+\mathrm{H}]^{+}\right)$; HRESIMS (m/e): calcd. for $\mathrm{C}_{65} \mathrm{H}_{54} \mathrm{~N}_{5} \mathrm{O}_{5}\left([\mathrm{M}+\mathrm{H}]^{+}\right)$952.4227, found 952.4224; UV-Vis $\left(\mathrm{CH}_{2} \mathrm{Cl}_{2}\right) \lambda_{\max } / \mathrm{nm}: 418,515,550,589,645$.

\section{Photooxygenation}

\section{Experiment Design}


The photooxygenation took place in an NMR tube with a sensitizer-drug complex dissolved in deuterated solvent $\left(2 \times 10^{-3}\right.$ to $\left.1.5 \times 10^{-2} \mathrm{M}\right)$. The NMR tube was placed in the light path $10 \mathrm{~cm}$ from the lamp, where the light was focused by the adjustable lens of the lamp house. During the photooxygenation, the NMR tube was open to air to keep the supply of oxygen and the reaction solution was maintained at room temperature by water-bath. It was found unnecessary to introduce an additional source of oxygen due to the small reaction scale.

The progress of the photooxygenation was monitored by NMR and GC and 1,3,5-trimethoxybenzene was added to the system as internal standard prior to the photoirradiation. Blank tests showed that 1,3,5-trimethoxybenzene does not react with singlet oxygen and remains intact under the photooxygenation conditions. The yields of photooxygenation products were determined by NMR integration of corresponding characteristic peaks referenced to that of the internal standard. On the other hand, the yields of the total releasable drugs (esters or amides) from the photooxygenation experiment were calculated by GC using internal standardization, based on the fact that all the dioxetane generated from the photooxygenation decomposed instantly at the injection temperature $\left(220{ }^{\circ} \mathrm{C}\right)$. In addition, the photooxygenation crude mixture was studied by Mass Spectrometry to confirm the proposed products or intermediates.

\section{General Procedure for Photooxygenation (GP-4)}

To a deuterated solution $(0.5 \mathrm{~mL})$ of a photosensitizer-drug complex $(3 \mu \mathrm{mol})$ in 
a $5 \mathrm{~mm}$ NMR tube was added the internal standard, 1,3,5-trimethoxybenzene $(0.5 \mathrm{mg}, 3$ $\mu \mathrm{mol})$. A filtered visible light $(\lambda>550 \mathrm{~nm}$ and $680 \mathrm{~nm}>\lambda>620 \mathrm{~nm}$ respectively for TPPAD or BPAD based complexes, $210 \mathrm{~W}$ output power) was shone onto the reaction solution. From time to time, the photoirradiation was interrupted and the NMR tube was capped and shaken vigorously before $0.5 \mu \mathrm{L}$ of the reaction mixture was removed and injected into GC for the quantification of released drug. The head pressure and the injection temperature of $\mathrm{GC}$ were set to $15 \mathrm{psi}$ and $220{ }^{\circ} \mathrm{C}$, respectively. Two temperature programs were employed: A. increasing from $80{ }^{\circ} \mathrm{C}$ (maintaining for $1 \mathrm{~min}$ ) to $220{ }^{\circ} \mathrm{C}$ at $10{ }^{\circ} \mathrm{C} / \mathrm{min}$; and $\mathrm{B}$. increasing from $40{ }^{\circ} \mathrm{C}$ (maintaining for $3 \mathrm{~min}$ ) to $220{ }^{\circ} \mathrm{C}$ at $10{ }^{\circ} \mathrm{C} / \mathrm{min}$. The retention times of 1,3,5-trimethoxybenzene for Program A and B are $10.00 \mathrm{~min}$ and 16.07 min, respectively. Program A was generally applied except for the photooxygenation of the $\mathbf{2 Z}$ and $\mathbf{2 E}$, where Program B was used. Simultaneously, the reaction mixture was characterized by ${ }^{1} \mathrm{H}$ (and/or ${ }^{1} \mathrm{H}-{ }^{1} \mathrm{H}$ COSY) NMR spectroscopy, before the photoirradiation was continued for the next period. Such photoirradiationcharacterization cycles were continued until all of the starting photosensitizer-drug complex was converted.

\subsection{Photooxygenation of $2 \mathrm{Z}$}

In $C_{6} D_{6}($ Table 1 , entry 1)

Following the general photooxygenation procedure (GP-4), $2 \mathrm{Z}(3.5 \mathrm{mg}, 4 \mu \mathrm{mol})$ and 1,3,5-trimethoxybenzene $(0.7 \mathrm{mg}, 4 \mu \mathrm{mol})$ were dissolved in $0.5 \mathrm{~mL} \mathrm{C}_{6} \mathrm{D}_{6}$. Photoirradiation of the reaction solution for 4 min yielded (by NMR) dioxetane $(22 \%)$, 
ethyl butyrate (71\%) and the photosensitizer fragment (65\%). The GC yield of total releasable ethyl butyrate was $94 \%$.

In $\mathrm{CDCl}_{3}$ (Table 1, entry 2)

Following the general photooxygenation procedure (GP-4), 2Z (1.4 mg, 1.6 $\mu \mathrm{mol})$ and 1,3,5-trimethoxybenzene $(0.5 \mathrm{mg}, 3 \mu \mathrm{mol})$ were dissolved in $0.5 \mathrm{~mL} \mathrm{CDCl}{ }_{3}$. Photoirradiation of the reaction solution for 3 min yielded dioxetane (70\%), ethyl butyrate (21\%), and the photosensitizer fragment (17\%). The GC yield of total releasable ethyl butyrate was quantitative. Attempts to isolate the dioxetane intermediate were unsuccessful as it decomposed on silica gel. Nevertheless, the photosensitizer fragment was obtained.

Photosensitizer fragment from the photooxygenation of TPPA based complexes

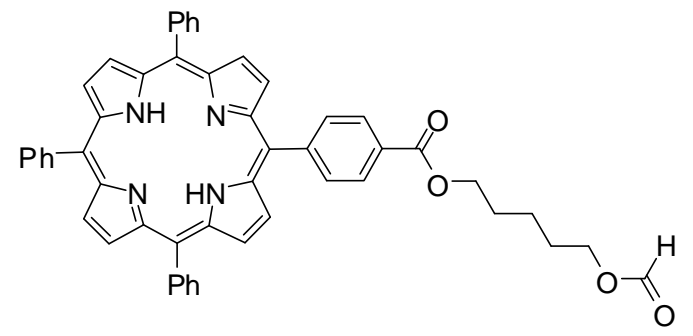

$\mathrm{R}_{f}\left(\right.$ silica- $\left.\mathrm{CH}_{2} \mathrm{Cl}_{2}\right)$ 0.26; ${ }^{1} \mathrm{H}-\mathrm{NMR}\left(300 \mathrm{MHz}, \mathrm{CDCl}_{3}\right): \delta=8.86-8.84(\mathrm{~m}, 6 \mathrm{H})$, $8.78(\mathrm{~d}, 2 \mathrm{H}, \mathrm{J}=4.7 \mathrm{~Hz}), 8.42(\mathrm{~d}, 2 \mathrm{H}, \mathrm{J}=7.8 \mathrm{~Hz}), 8.30(\mathrm{~d}, 2 \mathrm{H}, \mathrm{J}=7.8 \mathrm{~Hz}), 8.22-8.19(\mathrm{~m}$, $6 \mathrm{H}), 8.10\left(\mathrm{~s}, 1 \mathrm{H}, \mathrm{C}^{6}\right), 7.78-7.70(\mathrm{~m}, 9 \mathrm{H}), 4.52(\mathrm{t}, 2 \mathrm{H}, \mathrm{J}=6.6 \mathrm{~Hz}), 4.26(\mathrm{t}, 2 \mathrm{H}, \mathrm{J}=6.4 \mathrm{~Hz})$, 1.97-1.85 (m, 2H), 1.83-1.72 (m, 2H), 1.70-1.62 (m, 2H), -2.79 (s, 2H); LRESIMS (m/e): $773.3\left([\mathrm{M}+\mathrm{H}]^{+}\right)$; HRESIMS (m/e): calcd. for $\mathrm{C}_{51} \mathrm{H}_{41} \mathrm{~N}_{4} \mathrm{O}_{4}\left([\mathrm{M}+\mathrm{H}]^{+}\right)$773.3128, found 773.3140; UV-Vis $\left(\mathrm{CH}_{2} \mathrm{Cl}_{2}\right) \lambda_{\max } / \mathrm{nm}(\log \varepsilon): 418$ (5.76), 515 (4.28), 550 (3.96), 590 (3.84), $645(3.74)$.. 
Dioxetane (not isolated, data were obtained from a mixture sample)

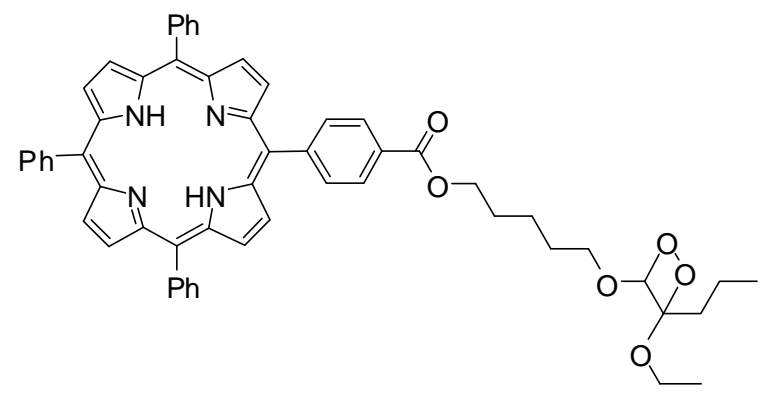

$\mathrm{R}_{f}\left(\right.$ silica- $\left.\mathrm{CH}_{2} \mathrm{Cl}_{2}\right)$ 0.37; ${ }^{1} \mathrm{H}-\mathrm{NMR}\left(300 \mathrm{MHz}, \mathrm{CDCl}_{3}\right.$ ): $\delta=8.85-8.83(\mathrm{~m}, 6 \mathrm{H})$, $8.77(\mathrm{~d}, 2 \mathrm{H}, \mathrm{J}=4.8 \mathrm{~Hz}), 8.42(\mathrm{~d}, 2 \mathrm{H}, \mathrm{J}=8.1 \mathrm{~Hz}), 8.29(\mathrm{~d}, 2 \mathrm{H}, \mathrm{J}=8.1 \mathrm{~Hz}), 8.22-8.19(\mathrm{~m}$, 6H), 7.78-7.70 (m, 9H), $5.86\left(\mathrm{~s}, 1 \mathrm{H}, \mathrm{B}^{6}\right), 4.51(\mathrm{t}, 2 \mathrm{H}, \mathrm{J}=6.4 \mathrm{~Hz}), 4.35-4.28(\mathrm{~m}, 2 \mathrm{H})$, 3.80-3.62 (m, 2H), 1.95-1.65 (m, 8H), 1.52-1.48 (m, 2H), $1.25(\mathrm{t}, 2 \mathrm{H}, \mathrm{J}=7.1 \mathrm{~Hz}), 0.94(\mathrm{t}$, 2H, J = 7.4 Hz), -2.80 (s, 2H); LRESIMS (m/e): $889.1\left([\mathrm{M}+\mathrm{H}]^{+}\right)$; HRESIMS (m/e): calcd. for $\mathrm{C}_{57} \mathrm{H}_{53} \mathrm{~N}_{4} \mathrm{O}_{6}\left([\mathrm{M}+\mathrm{H}]^{+}\right)$889.3965, found 889.3966.

In $\mathrm{CDCl}_{3}: \mathrm{CD}_{3} \mathrm{OD}=4: 1$ mixed solvent (Table 1, entry 3)

Following the general photooxygenation procedure (GP-4), 2Z (1.6 mg, 1.8 $\mu \mathrm{mol})$ and 1,3,5-trimethoxybenzene $(0.5 \mathrm{mg}, 3 \mu \mathrm{mol})$ were dissolved in $0.5 \mathrm{~mL}$ $\mathrm{CDCl}_{3}: \mathrm{CD}_{3} \mathrm{OD}=4: 1$ mixed solvent. Photoirradiation of the reaction solution for $3 \mathrm{~min}$ yielded dioxetane (73\%), ethyl butyrate (17\%) and the photosensitizer fragment (20\%). The GC yield of total releasable ethyl butyrate was quantitative.

In $\mathrm{CD}_{3} \mathrm{C}(\mathrm{O}) \mathrm{CD}_{3}$ (Table 1, entry 4)

Following the general photooxygenation procedure (GP-4), $2 Z$ ( $2 \mathrm{mg}, 2.2 \mu \mathrm{mol}$ ) and 1,3,5-trimethoxybenzene $(0.3 \mathrm{mg}, 2 \mu \mathrm{mol})$ were dissolved in $0.5 \mathrm{~mL} \mathrm{CD}{ }_{3} \mathrm{C}(\mathrm{O}) \mathrm{CD}_{3}$. 
Photoirradiation of the reaction solution for 8 min yielded dioxetane (38\%), ethyl butyrate (56\%) and the photosensitizer fragment (61\%). The GC yield of total releasable ethyl butyrate was quantitative.

In $\mathrm{CD}_{3} \mathrm{C}(\mathrm{O}) \mathrm{CD}_{3}$ with $\mathrm{DABCO}$ adduct (Table 1, entry 5)

Following the general photooxygenation procedure (GP-4), $2 Z$ ( $2 \mathrm{mg}, 2.2 \mu \mathrm{mol}$ ) and 1,3,5-trimethoxybenzene $(0.7 \mathrm{mg}, 4 \mu \mathrm{mol})$ were dissolved in $0.5 \mathrm{~mL} \mathrm{CD}{ }_{3} \mathrm{C}(\mathrm{O}) \mathrm{CD}_{3}$ and 1,4-diazabicyclo[2.2.2]octane (DABCO, $1.5 \mathrm{mg}, 13.4 \mathrm{mmol}$ ) was added to the reaction solution before it was illuminated for $1 \mathrm{~h}$ to yield ethyl butyrate (quantitative) and the photosensitizer fragment (quantitative). The GC yield of total releasable ethyl butyrate was quantitative.

\subsection{Photooxygenation of 3Z (Table 1, entry 6)}

Following the general photooxygenation procedure (GP-4), $3 Z$ (1.5 mg, 1.5 $\mu \mathrm{mol})$ and 1,3,5-trimethoxybenzene $(0.3 \mathrm{mg}, 2 \mu \mathrm{mol})$ were dissolved in $0.5 \mathrm{~mL} \mathrm{C}_{6} \mathrm{D}_{6}$. Photoirradiation of the reaction solution for 1 min yielded dioxetane (72\%), the photosensitizer fragment (28\%) and ethyl benzoate (12\%). The GC yield of total releasable ethyl benzoate was $91 \%$.

Dioxetane (not isolated, data were obtained from a mixture sample) 


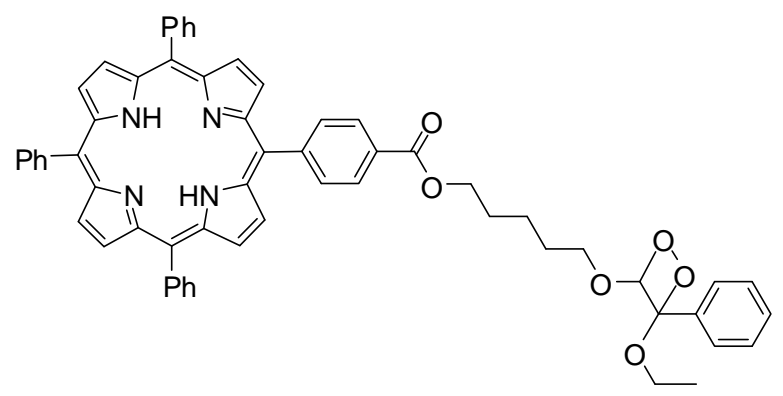

${ }^{1} \mathrm{H}-\mathrm{NMR}\left(400 \mathrm{MHz}, \mathrm{C}_{6} \mathrm{D}_{6}\right): \delta=8.95(\mathrm{~d}, 2 \mathrm{H}, \mathrm{J}=4.8 \mathrm{~Hz}), 8.92(\mathrm{~s}, 4 \mathrm{H}), 8.82(\mathrm{~d}$, 2H, J = 4.7 Hz), $8.49(\mathrm{~d}, 2 \mathrm{H}, \mathrm{J}=8.1 \mathrm{~Hz}), 8.13-8.04(\mathrm{~m}, 8 \mathrm{H}), 7.89(\mathrm{~d}, 2 \mathrm{H}, \mathrm{J}=7.2 \mathrm{~Hz})$, 7.49-7.43 (m, 9H), 7.17-7.03 (m, 3H), $5.85(\mathrm{~s}, 1 \mathrm{H}), 4.54-4.31(\mathrm{~m}, 2 \mathrm{H}), 4.33(\mathrm{t}, 2 \mathrm{H}, \mathrm{J}=$ $6.5 \mathrm{~Hz}), 3.36-3.17$ (m, 2H), 1.56 (m, 2H), 1.38-1.33 (m, 4H), 1.27 (t, 3H, J = 7.0 Hz), 2.14 (s, 2H); $\operatorname{ESIMS~(m/e):~} 923.1\left([\mathrm{M}+\mathrm{H}]^{+}\right)$.

\subsection{Photooxygenation of $\mathbf{4 Z}$ (Table 1, entry 7)}

Following the general photooxygenation procedure (GP-4), $4 \mathrm{Z}$ (4.2 mg, $5 \mu \mathrm{mol}$ ) and 1,3,5-trimethoxybenzene $(0.6 \mathrm{mg}, 4 \mu \mathrm{mol})$ were dissolved in $0.5 \mathrm{~mL} \mathrm{~d}_{6}$-acetone. Photoirradiation of the reaction solution for $3 \mathrm{~min}$ yielded dioxetane $(35 \%), \delta$ valerolactone $(59 \%)$ and the photosensitizer fragment (46\%). The GC yield of total releasable $\delta$-valerolactone was $90 \%$.

Dioxetane (not isolated, data were obtained from a mixture sample)

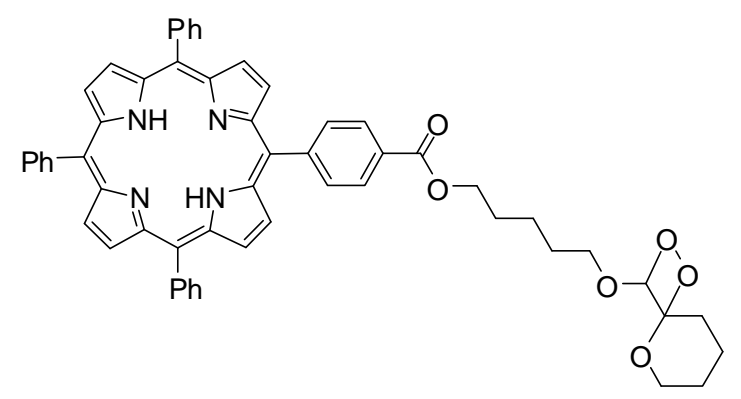


${ }^{1} \mathrm{H}-\mathrm{NMR}\left(400 \mathrm{MHz}, \mathrm{d}_{6}\right.$-acetone): $\delta=8.88(\mathrm{~m}, 8 \mathrm{H}), 8.47(\mathrm{~d}, 2 \mathrm{H}, \mathrm{J}=8.0 \mathrm{~Hz})$, $8.38(\mathrm{~d}, 2 \mathrm{H}, \mathrm{J}=8.1 \mathrm{~Hz}), 8.27-8.24(\mathrm{~m}, 6 \mathrm{H}), 7.86-7.80(\mathrm{~m}, 9 \mathrm{H}), 5.84(\mathrm{~s}, 1 \mathrm{H}), 4.52(\mathrm{t}, 2 \mathrm{H}$, $\mathrm{J}=6.5 \mathrm{~Hz}), 3.97-3.81(\mathrm{~m}, 4 \mathrm{H}), 2.02-1.67(\mathrm{~m}, 12 \mathrm{H}),-2.74(\mathrm{~s}, 2 \mathrm{H})$; LRESIMS $(\mathrm{m} / \mathrm{e})$ : $873.5\left([\mathrm{M}+\mathrm{H}]^{+}\right)$; HRESIMS (m/e): calcd. for $\mathrm{C}_{56} \mathrm{H}_{49} \mathrm{~N}_{4} \mathrm{O}_{6}\left([\mathrm{M}+\mathrm{H}]^{+}\right)$873.3652, found 873.3654 .

\subsection{Photooxygenation of $5 \mathrm{Z}$ (Table 1, entry 8)}

Following the general photooxygenation procedure (GP-4), $5 \mathrm{Z}$ (7.2 mg, 7.5 $\mu \mathrm{mol})$ and 1,3,5-trimethoxybenzene $(2.7 \mathrm{mg}, 16 \mu \mathrm{mol})$ were dissolved in $0.5 \mathrm{~mL} \mathrm{C}_{6} \mathrm{D}_{6}$. Photoirradiation of the reaction solution for 2.5 min yielded dioxetane (69\%), ibuprofen methyl ester $(20 \%)$ and the photosensitizer fragment $(23 \%)$. The GC yield of total releasable ibuprofen methyl ester was quantitative.

Dioxetane (not isolated, 2 pairs of diastereomers showed 2 sets of ${ }^{1}$ H-NMR signals at 1:1 ratio)

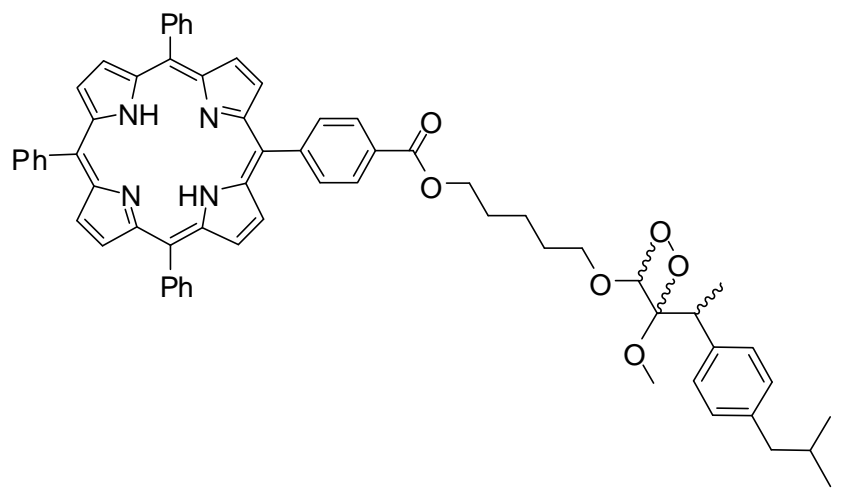

${ }^{1} \mathrm{H}-\mathrm{NMR}\left(400 \mathrm{MHz}, \mathrm{C}_{6} \mathrm{D}_{6}\right.$, for each set, one proton was integrated as $\left.0.5 \mathrm{H}\right): \delta=$ 8.96-8.95 (m, 2H), 8.93 (s, 4H), 8.84-8.81 (m, 2H), $8.50(\mathrm{~d}, 2 \mathrm{H}, \mathrm{J}=8.1 \mathrm{~Hz}), 8.13-8.06$ (m, 8H), 7.49-7.43 (m, 9H), 7.42-7.40 (m, 2H), $7.01(\mathrm{~d}, 2 \mathrm{H}, \mathrm{J}=8.0 \mathrm{~Hz}), 5.77(\mathrm{~s}, 0.5 \mathrm{H})$, 
$5.74(\mathrm{~s}, 0.5 \mathrm{H}), 4.32(\mathrm{t}, 2 \mathrm{H}, \mathrm{J}=6.5 \mathrm{~Hz}), 4.11(\mathrm{~s}, 3 \times 0.5 \mathrm{H})$, ), $4.02(\mathrm{~s}, 3 \mathrm{x} 0.5 \mathrm{H}), 3.54-3.12$ $(\mathrm{m}, 2 \mathrm{H}), 3.08-3.00(\mathrm{~m}, 0.5 \mathrm{H}), 2.88-2.80(\mathrm{~m}, 0.5 \mathrm{H}), 2.35(\mathrm{~d}, 2 \mathrm{x} 0.5 \mathrm{H}, \mathrm{J}=7.2 \mathrm{~Hz}), 2.29(\mathrm{~d}$, $2 \times 0.5 \mathrm{H}, \mathrm{J}=7.2 \mathrm{~Hz}), 1.78-1.29(\mathrm{~m}, 7 \mathrm{H}), 1.36\left(\mathrm{~d}, 3 \mathrm{H}, \mathrm{J}=7.2 \mathrm{~Hz}, \mathrm{~B}^{16}\right), 0.82(\mathrm{~d}, 6 \mathrm{x} 0.5 \mathrm{H}, \mathrm{J}$ $=6.4 \mathrm{~Hz}), 0.77(\mathrm{~d}, 6 \mathrm{x} 0.5 \mathrm{H}, \mathrm{J}=6.6 \mathrm{~Hz}),-2.14(\mathrm{~s}, 2 \mathrm{H})$; LRESIMS (m/e): $993.1\left([\mathrm{M}+\mathrm{H}]^{+}\right)$; HRESIMS (m/e): calcd. for $\mathrm{C}_{65} \mathrm{H}_{61} \mathrm{~N}_{4} \mathrm{O}_{6}\left([\mathrm{M}+\mathrm{H}]^{+}\right)$993.4591, found 993.4594 .

\subsection{Photooxygenation of 6Z (Table 1, entry 9)}

Following the general photooxygenation procedure (GP-4), $6 \mathrm{Z}$ ( $2 \mathrm{mg}, 2 \mu \mathrm{mol})$ and 1,3,5-trimethoxybenzene $(0.5 \mathrm{mg}, 3 \mu \mathrm{mol})$ were dissolved in $0.5 \mathrm{~mL} \mathrm{C}_{6} \mathrm{D}_{6}$. Photoirradiation of the reaction solution for 6 min yielded dioxetane (85\%), ibuprofen methyl ester (9\%) and the photosensitizer fragment (5\%). The GC yield of total releasable ibuprofen methyl ester was quantitative.

Dioxetane (not isolated, data were obtained from a mixture sample)

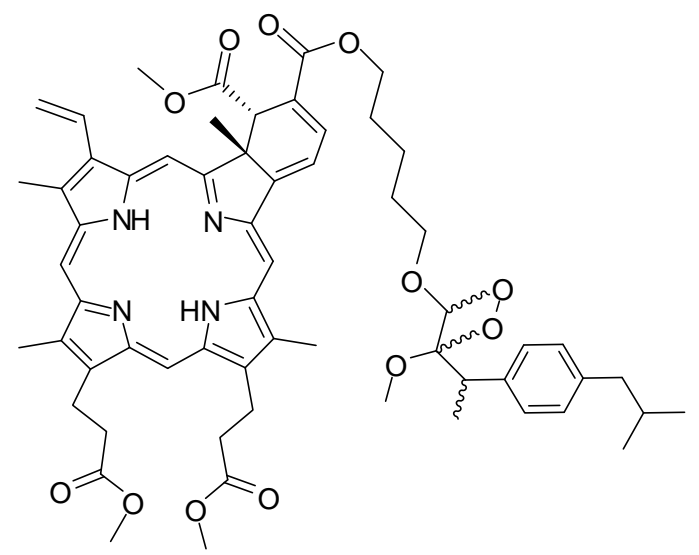

LRESIMS (m/e): $1053.2\left([\mathrm{M}+\mathrm{H}]^{+}\right)$; HRESIMS (m/e): calcd. for $\mathrm{C}_{61} \mathrm{H}_{73} \mathrm{~N}_{4} \mathrm{O}_{12}$ $\left([\mathrm{M}+\mathrm{H}]^{+}\right) 1053.5225$, found 1053.5228 . 


\subsection{Photooxygenation of $7 \mathrm{Z}$ (Table 1, entry 10)}

Following the general photooxygenation procedure (GP-4), $7 \mathrm{Z}(3.7 \mathrm{mg}, 3.8$ $\mu \mathrm{mol})$ and 1,3,5-trimethoxybenzene $(0.8 \mathrm{mg}, 4.5 \mu \mathrm{mol})$ were dissolved in $0.5 \mathrm{~mL} \mathrm{C}_{6} \mathrm{D}_{6}$. Photoirradiation of the reaction solution for 7 min yielded dioxetane (12\%), naproxen methyl ester (76\%) and the photosensitizer fragment (76\%). The GC yield of total releasable naproxen methyl ester was quantitative.

Dioxetane (not isolated, data were obtained from a mixture sample)

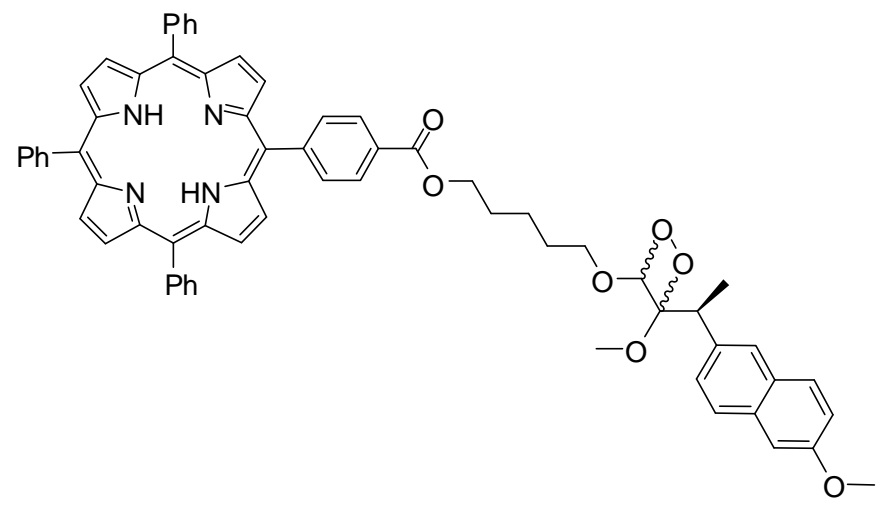

LRESIMS (m/e): $1017.0\left([\mathrm{M}+\mathrm{H}]^{+}\right)$; HRESIMS (m/e): calcd. for $\mathrm{C}_{66} \mathrm{H}_{57} \mathrm{~N}_{4} \mathrm{O}_{7}$ $\left([\mathrm{M}+\mathrm{H}]^{+}\right)$1017.4227, found 1017.4225.

\subsection{Photooxygenation of $\mathbf{8 Z}$ (Table 1, entry 11)}

Following the general photooxygenation procedure (GP-4), 8Z (3.7 mg, 3.6 $\mu \mathrm{mol})$ and 1,3,5-trimethoxybenzene $(0.7 \mathrm{mg}, 4 \mu \mathrm{mol})$ were dissolved in $0.5 \mathrm{~mL} \mathrm{C}_{6} \mathrm{D}_{6}$. Photoirradiation of the reaction solution for 3 min yielded dioxetane (27\%) and naproxen methyl ester (70\%). The GC yield of total releasable naproxen methyl ester was $86 \%$. 
Dioxetane (not isolated, data were obtained from a mixture sample)

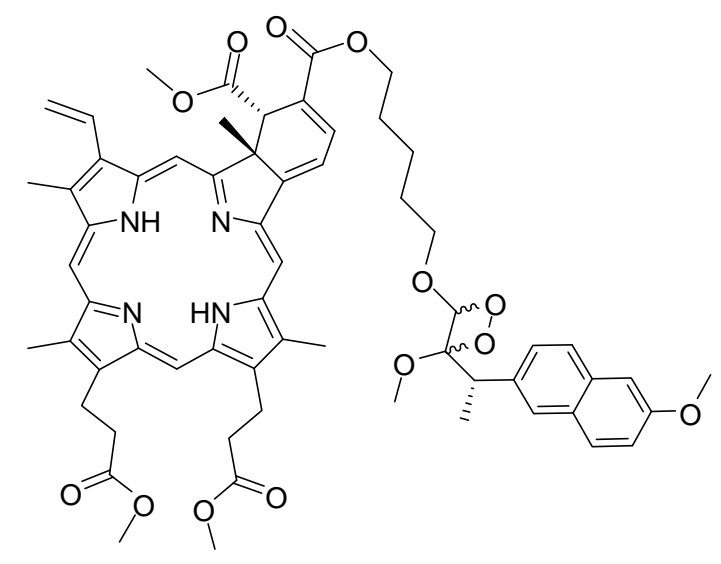

LRESIMS (m/e): $1077.2\left([\mathrm{M}+\mathrm{H}]^{+}\right)$; HRESIMS (m/e): calcd. for $\mathrm{C}_{62} \mathrm{H}_{69} \mathrm{~N}_{4} \mathrm{O}_{13}$ $\left([\mathrm{M}+\mathrm{H}]^{+}\right)$1077.4861, found 1077.4874.

3.8 Photooxygenation of 2E (Table 1, entry 12)

Following the general photooxygenation procedure (GP-4), 2E (0.85 mg, $1 \mu \mathrm{mol})$ and 1,3,5-trimethoxybenzene $(0.3 \mathrm{mg}, 2 \mu \mathrm{mol})$ were dissolved in $0.5 \mathrm{~mL} \mathrm{CDCl}_{3}$. Photoirradiation of the reaction solution for $1 \mathrm{~min}$ yielded hydroperoxide Z-isomer ("ene" product, 29\%), hydroperoxide E-isomer ("ene" product, 27\%) and dioxetane (37\%). The GC yield of total releasable ethyl butyrate was 33\%.

\subsection{Photooxygenation of 9 (Table 1, entry 13)}

Following the general photooxygenation procedure (GP-4), 9 (3.6 mg, $3.8 \mu \mathrm{mol}$, $\mathrm{Z}: \mathrm{E}=4: 1)$ and 1,3,5-trimethoxybenzene $(1.2 \mathrm{mg}, 8 \mu \mathrm{mol})$ were dissolved in $0.5 \mathrm{~mL} \mathrm{C}_{6} \mathrm{D}_{6}$. 
Photoirradiation of the reaction solution for 5 min yielded $\mathrm{N}$-methylbenzanilide (96\%) and the photosensitizer fragment $(96 \%)$. The GC yield of total releasable Nmethylbenzanilide was $88 \%$.

4 Selective NMR Spectra 


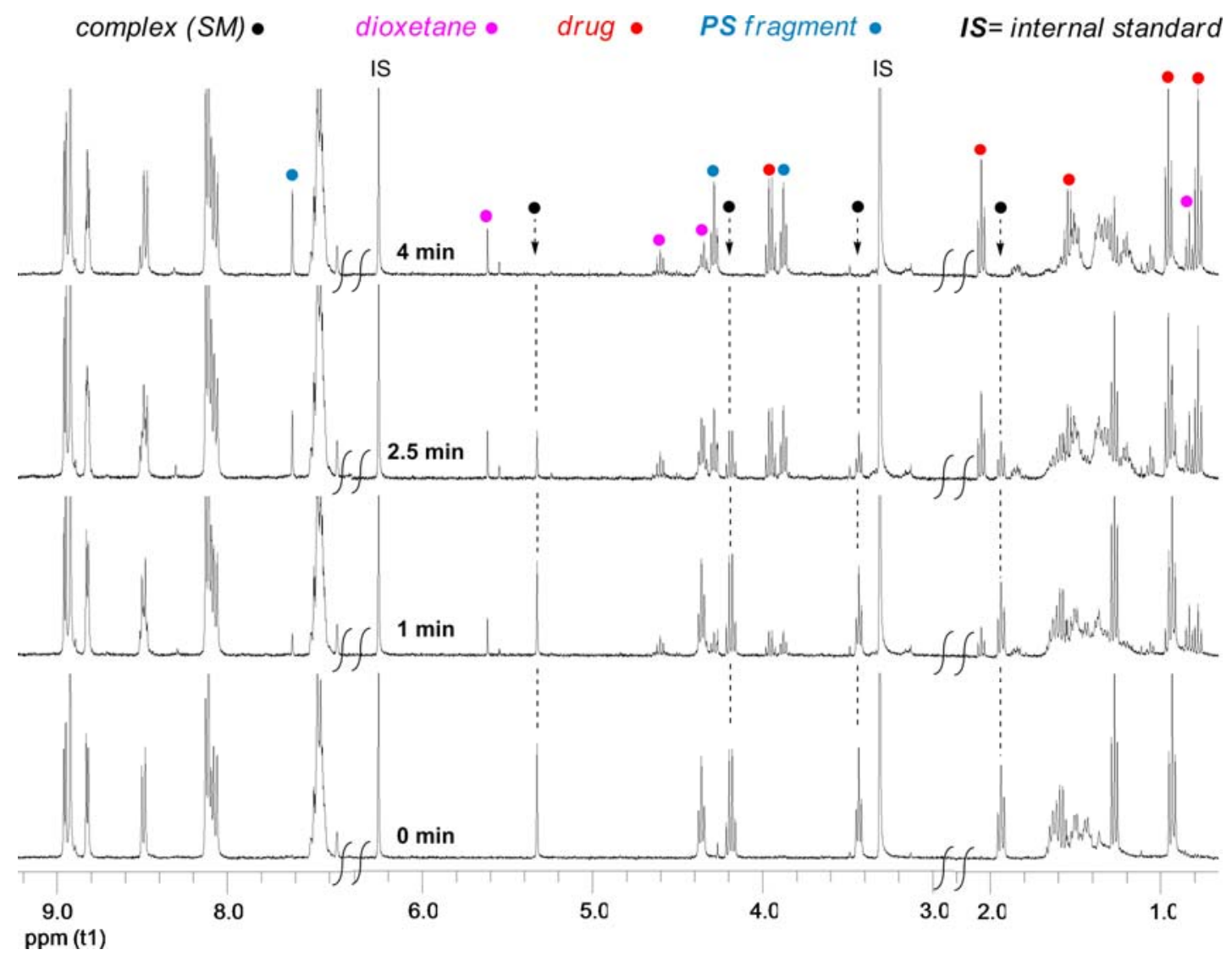

Figure 1 Photooxygenation of $\mathbf{2 Z}$ in $\mathrm{C}_{6} \mathrm{D}_{6}(400 \mathrm{MHz})$ 


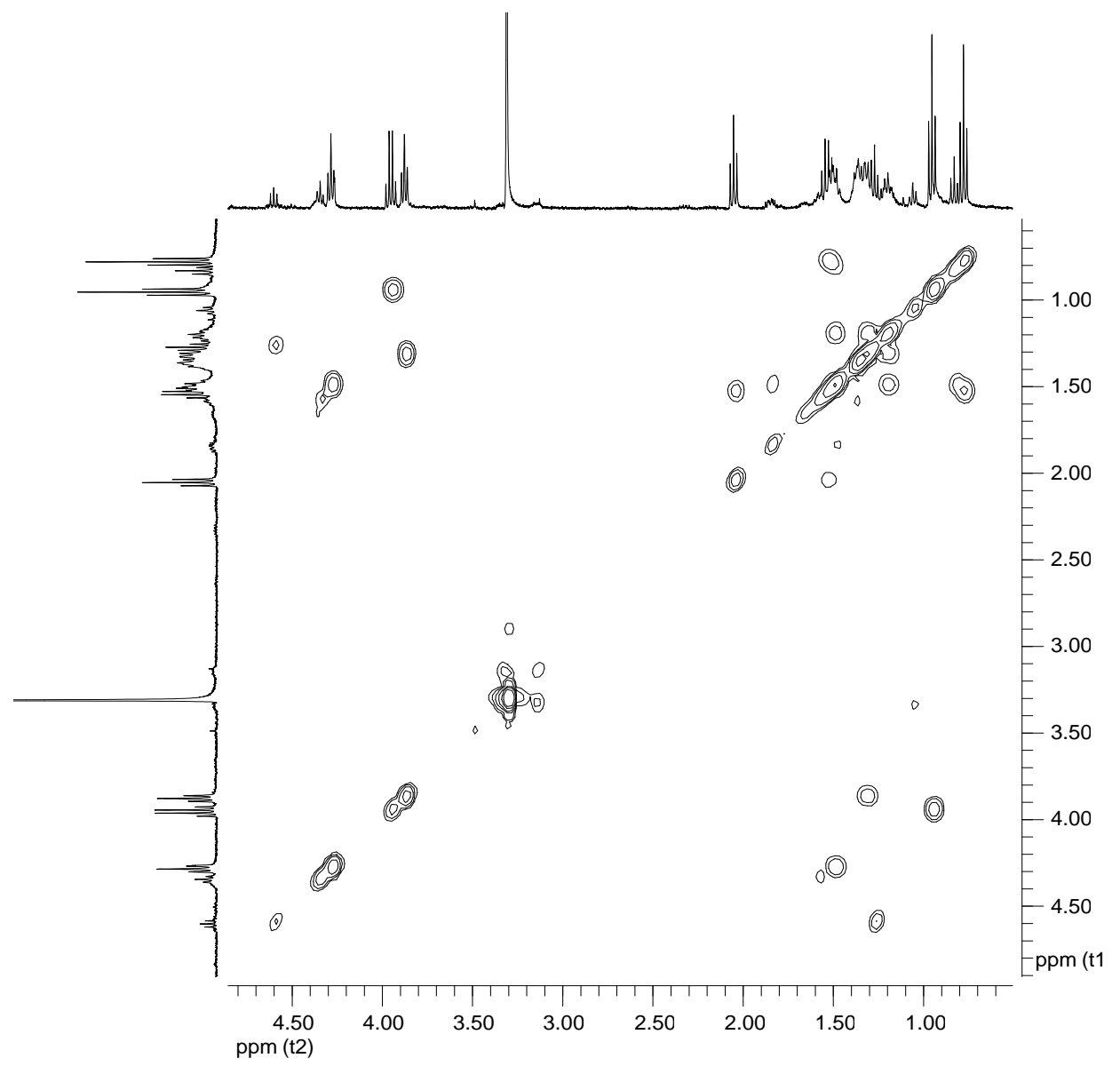

Figure 2 Photooxygenation crude mixture of $\mathbf{2 Z}\left(\mathrm{C}_{6} \mathrm{D}_{6}, 400 \mathrm{MHz}, 4 \mathrm{~min}\right.$ irradiation)

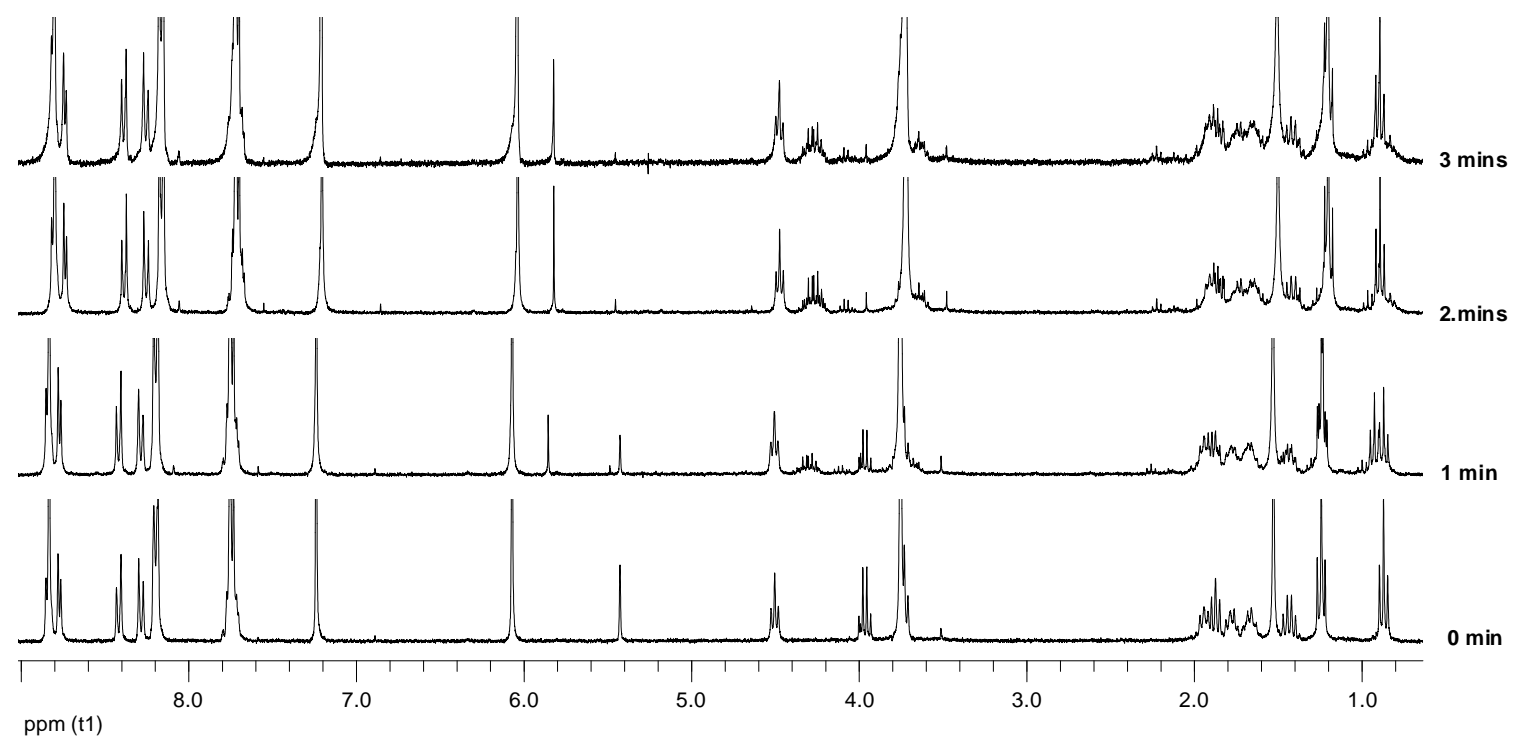

Figure 3 Photooxygenation of $\mathbf{2 Z}$ in $\mathrm{CDCl}_{3}(400 \mathrm{MHz})$ 


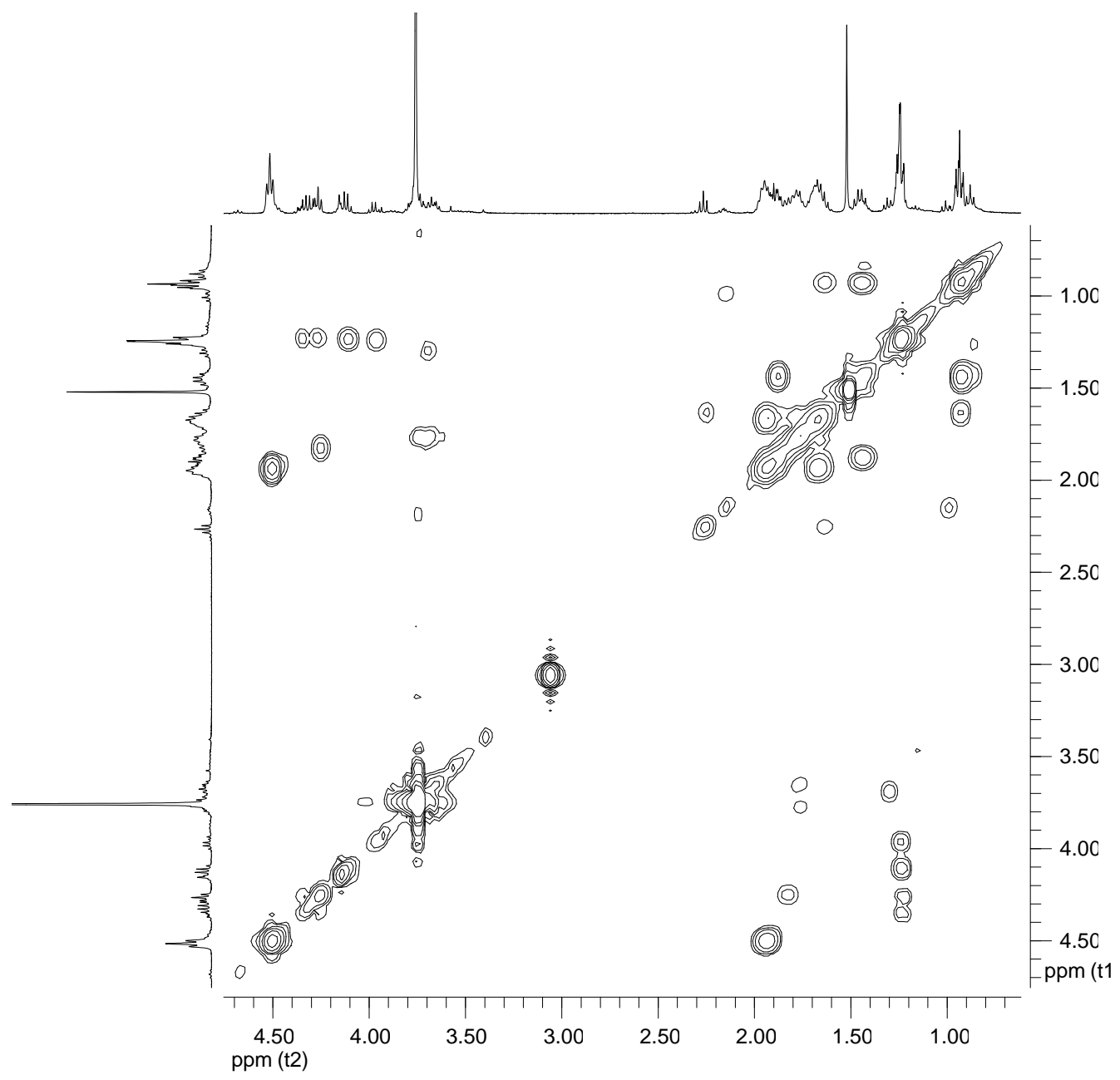

Figure 4 Photooxygenation crude mixture of $\mathbf{2 Z}\left(\mathrm{CDCl}_{3}, 400 \mathrm{MHz}, 2 \mathrm{~min}\right)$

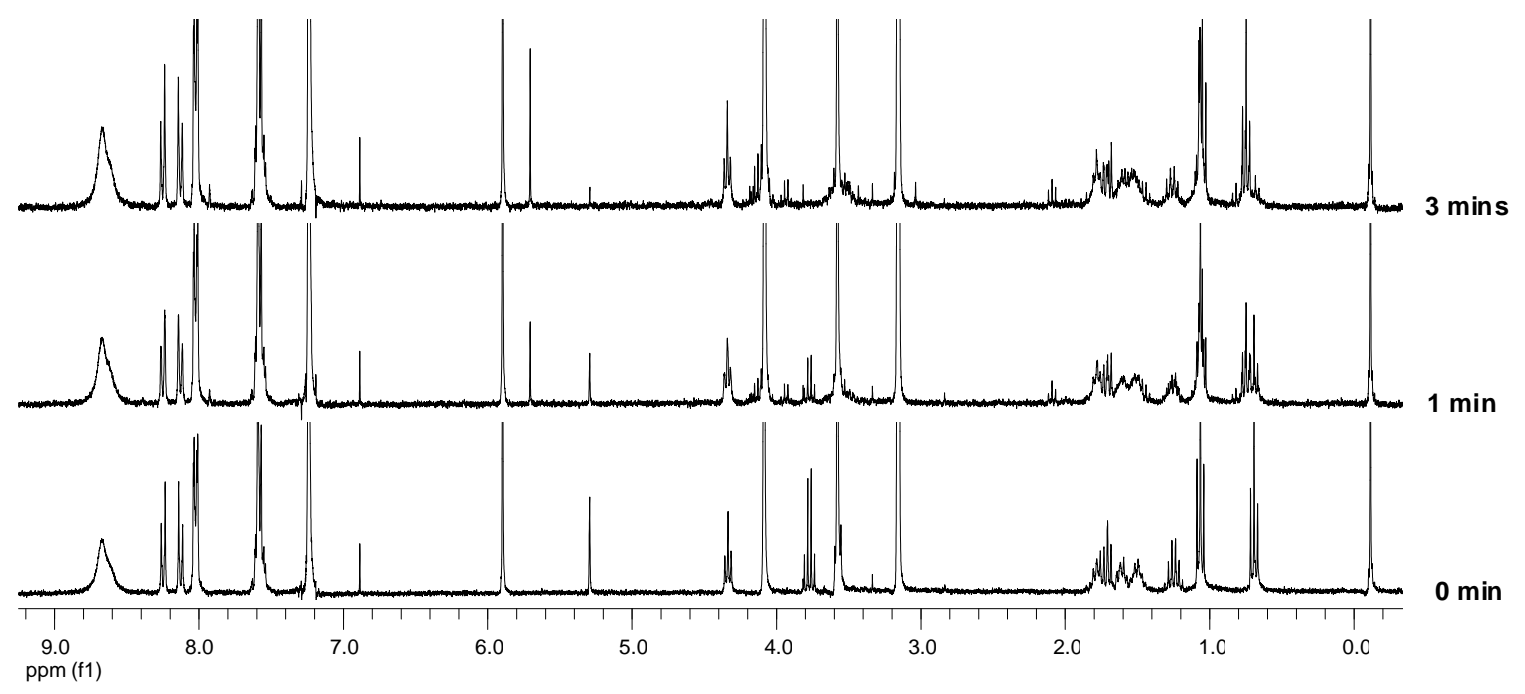

Figure 5 Photooxygenation of $2 \mathrm{Z}$ in mixed solvent $\left(\mathrm{CDCl}_{3}: \mathrm{MeOD}=4: 1,400 \mathrm{MHz}\right)$ 


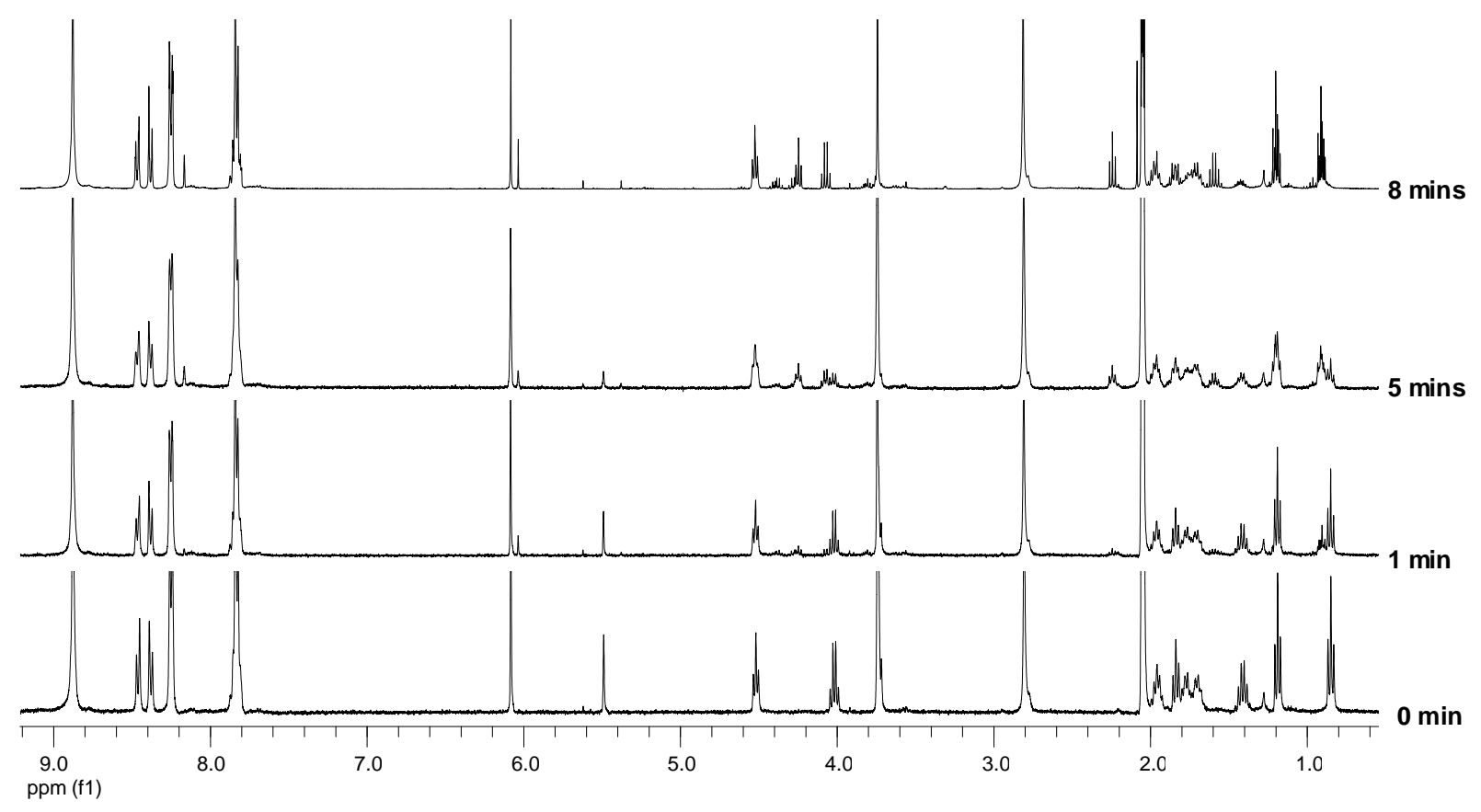

Figure 6 Photooxygenation of $\mathbf{2 Z}$ in $\mathrm{CD}_{3} \mathrm{C}(\mathrm{O}) \mathrm{CD}_{3}(400 \mathrm{MHz})$

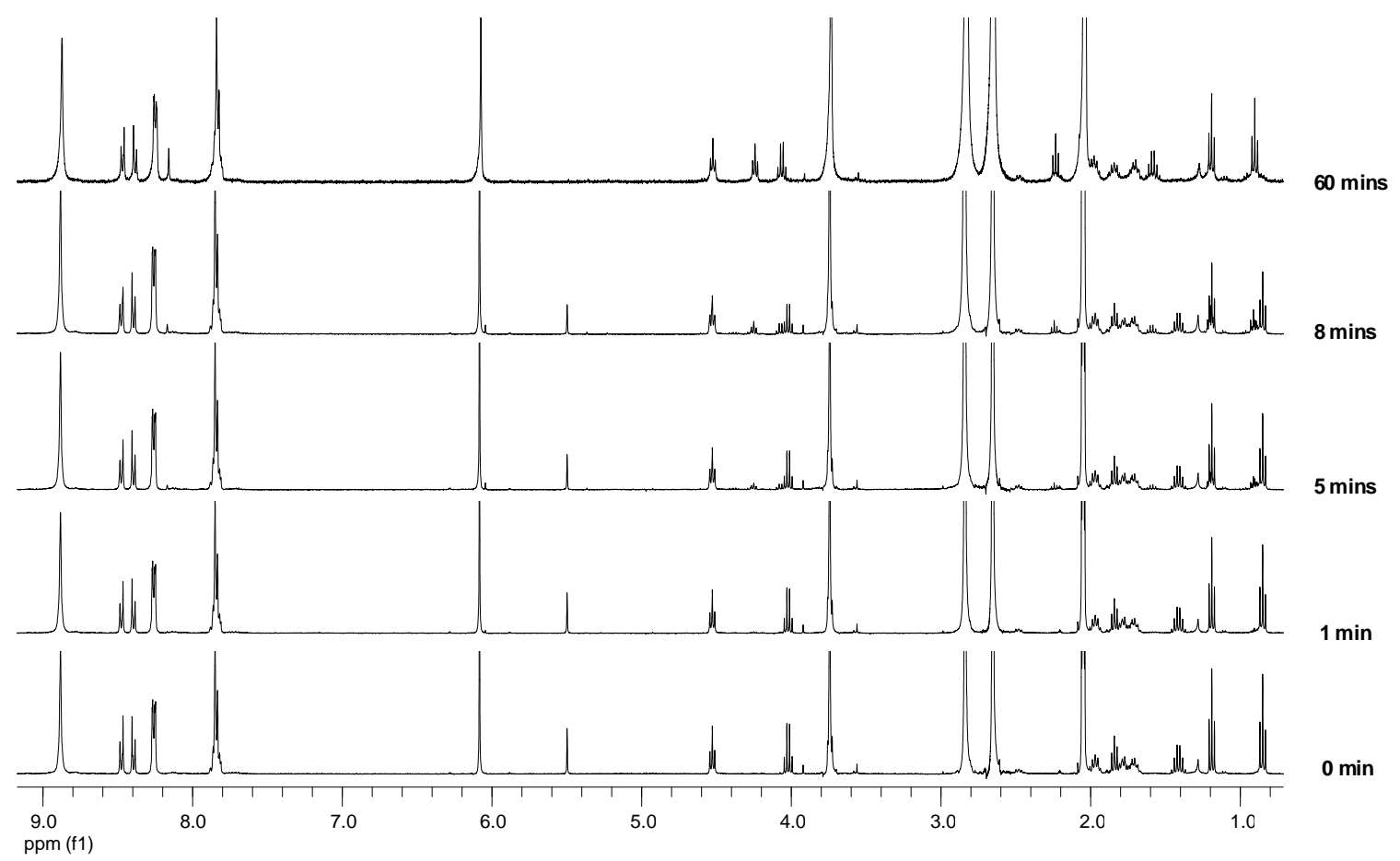

Figure 7 Photooxygenation of $\mathbf{2 Z}$ in $\mathrm{CD}_{3} \mathrm{C}(\mathrm{O}) \mathrm{CD}_{3}$ with $\mathrm{DABCO}$ adduct $(400 \mathrm{MHz})$ 


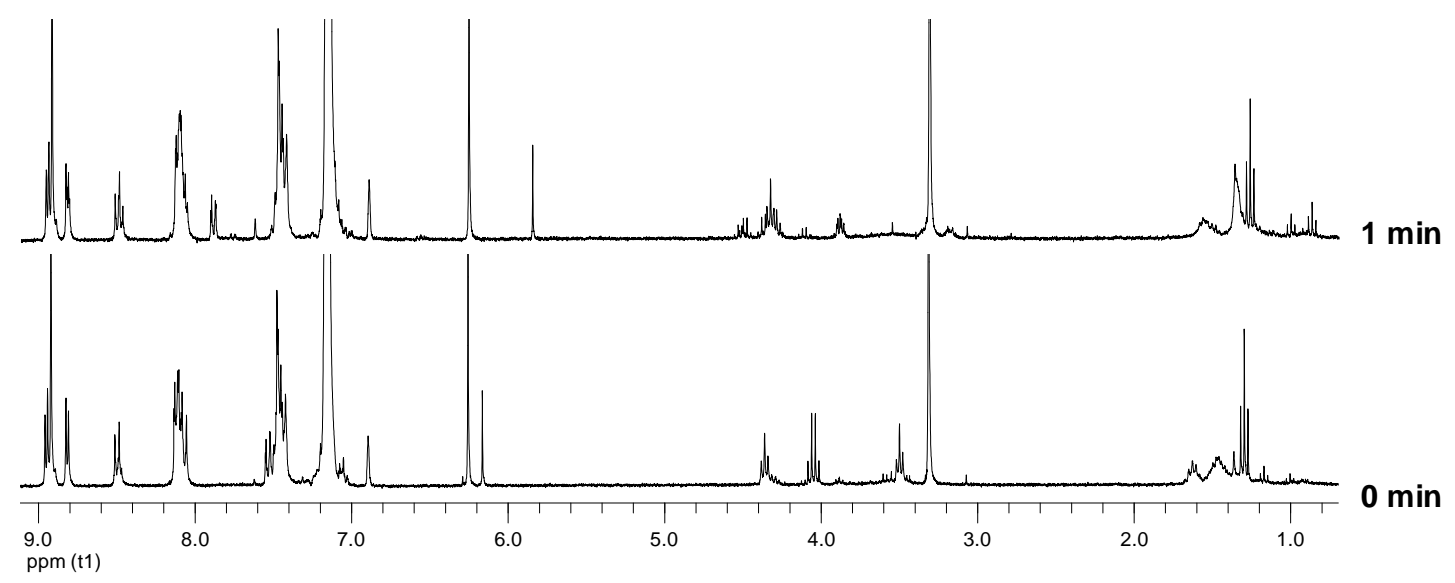

Figure 8 Photooxygenation of $\mathbf{3 Z}$ in $\mathrm{C}_{6} \mathrm{D}_{6}(300 \mathrm{MHz})$

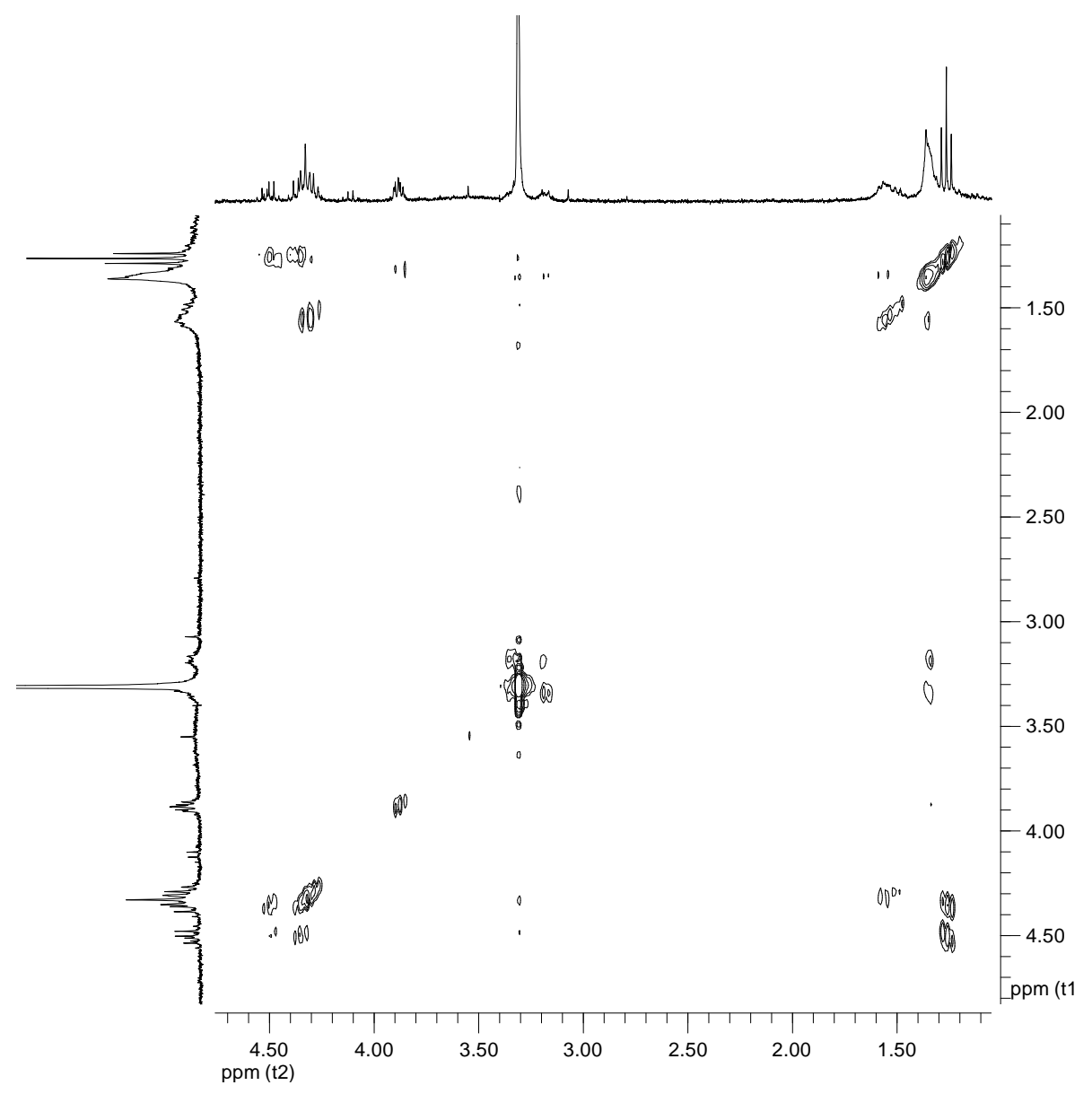

Figure 9 Photooxygenation crude mixture of $\mathbf{3 Z}\left(\mathrm{C}_{6} \mathrm{D}_{6}, 300 \mathrm{MHz}, 1 \mathrm{~min}\right)$ 


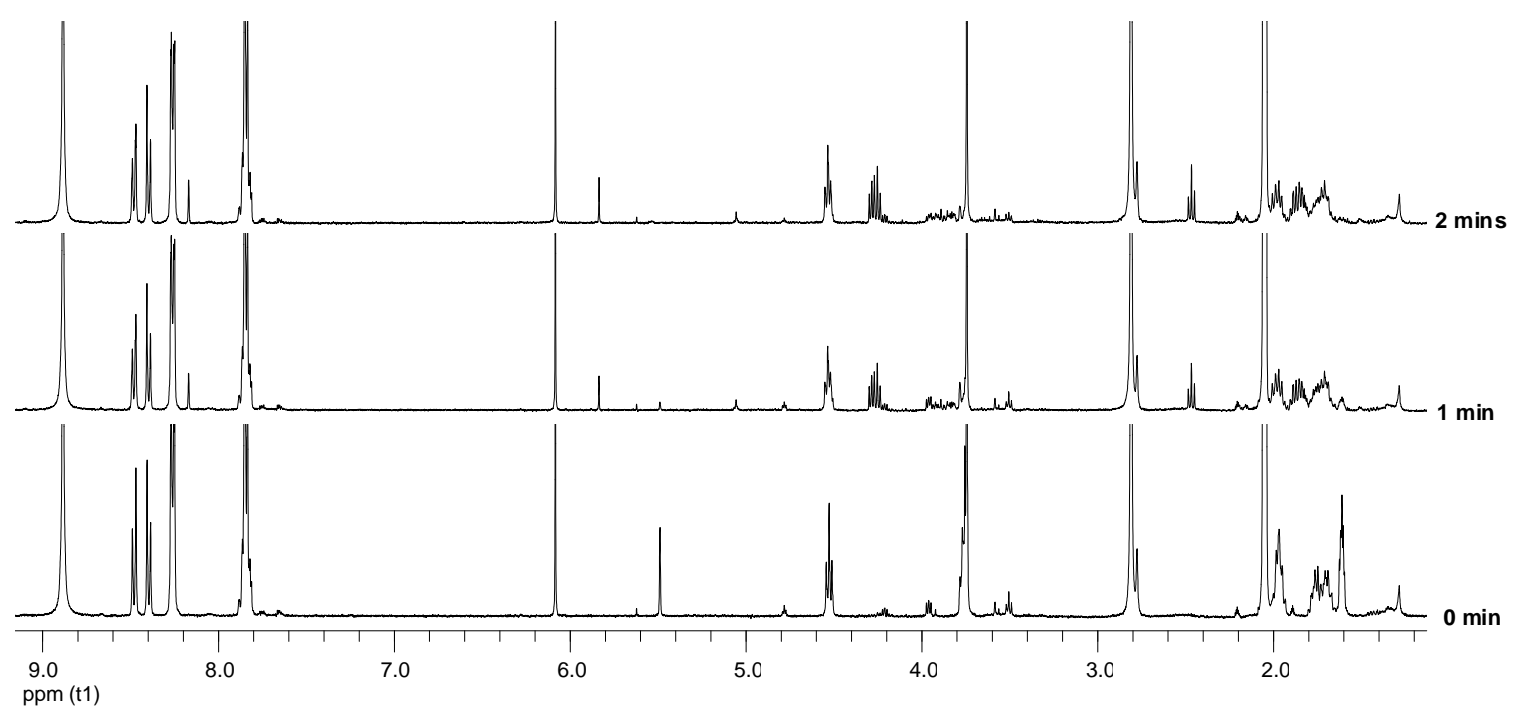

Figure 10 Photooxygenation of $\mathbf{4 Z}$ in $\mathrm{CD}_{3} \mathrm{C}(\mathrm{O}) \mathrm{CD}_{3}(400 \mathrm{MHz})$

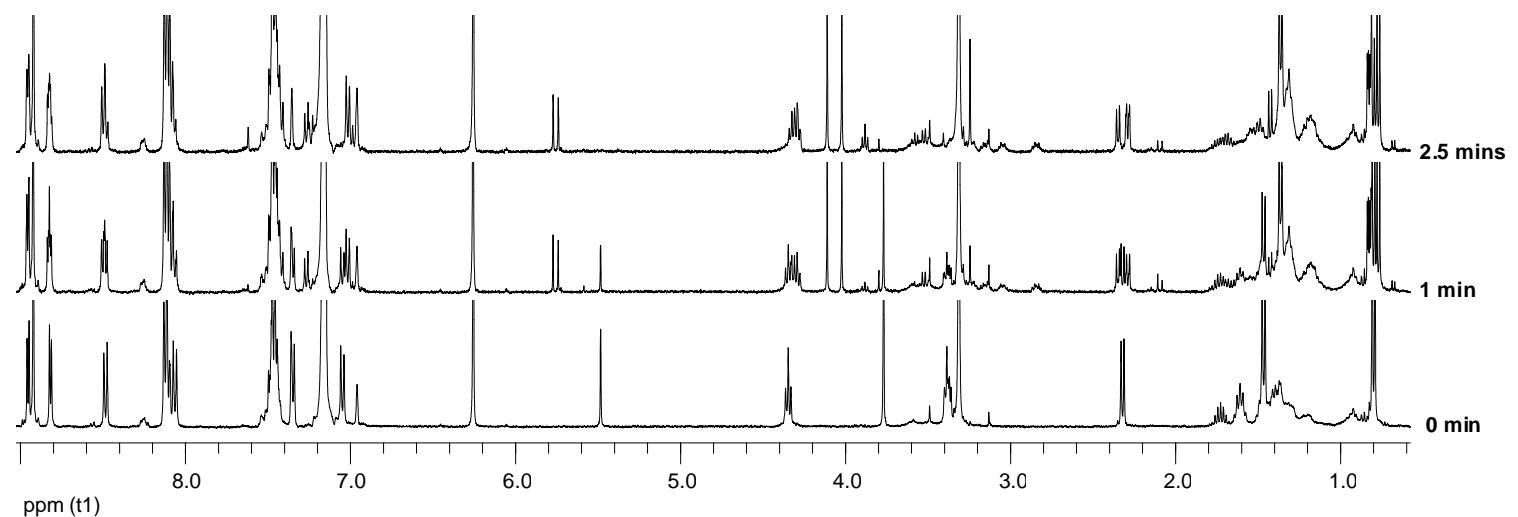

Figure 11 Photooxygenation of $5 \mathrm{Z}$ in $\mathrm{C}_{6} \mathrm{D}_{6}(400 \mathrm{MHz})$ 


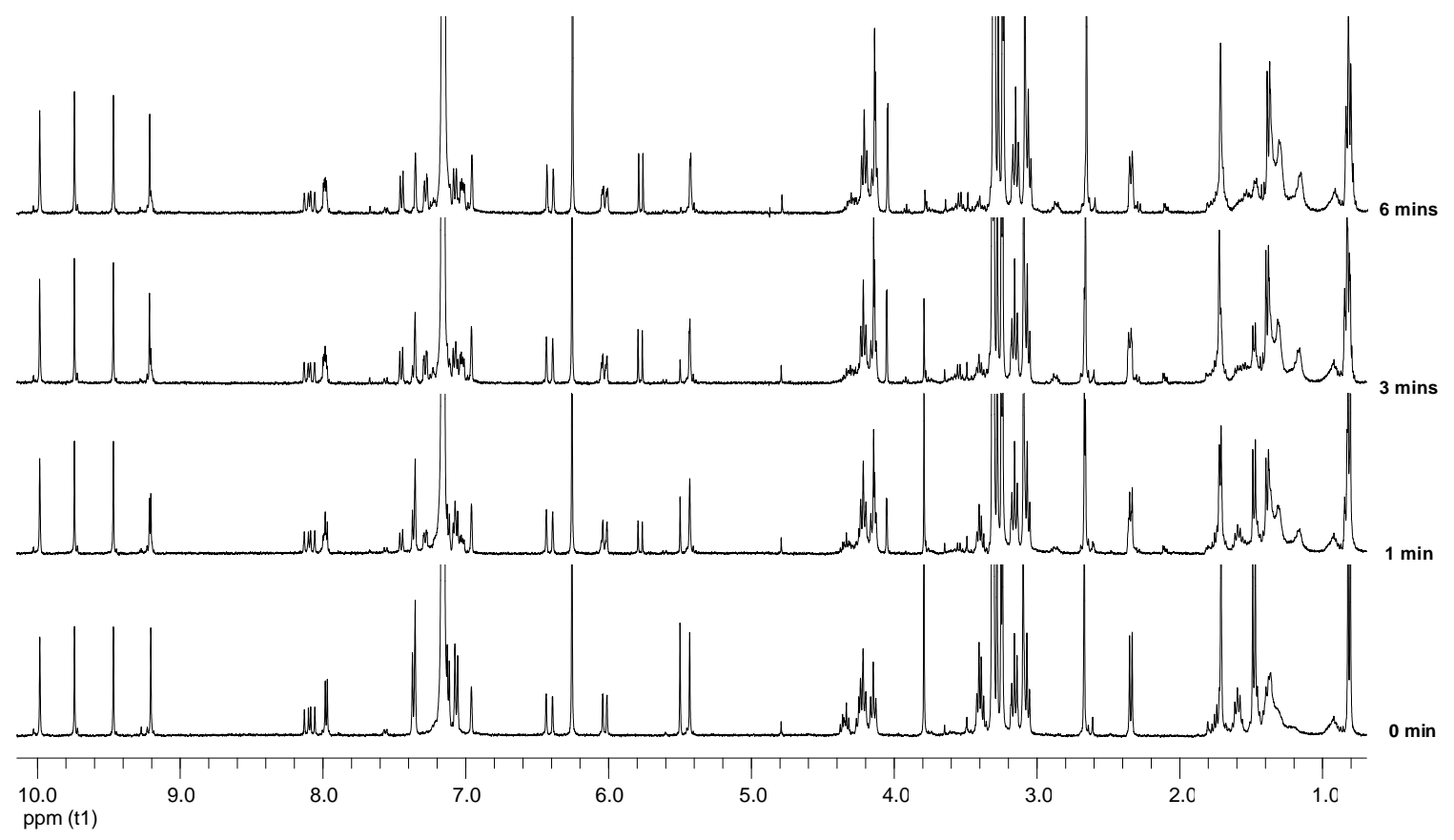

Figure 12 Photooxygenation of $\mathbf{6 Z}$ in $\mathrm{C}_{6} \mathrm{D}_{6}(400 \mathrm{MHz})$

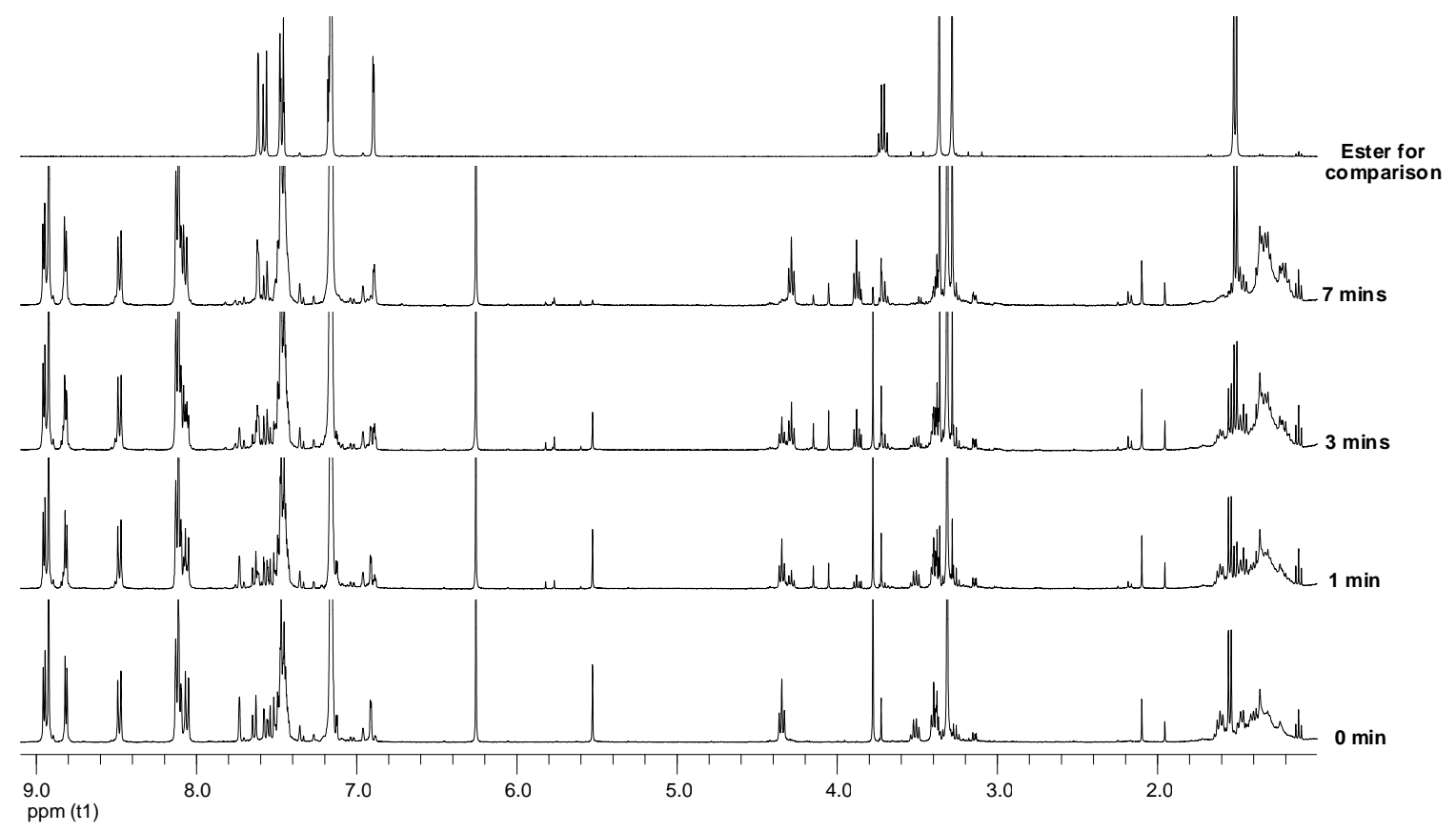

Figure 13 Photooxygenation of $7 \mathrm{Z}$ in $\mathrm{C}_{6} \mathrm{D}_{6}(400 \mathrm{MHz})$ 

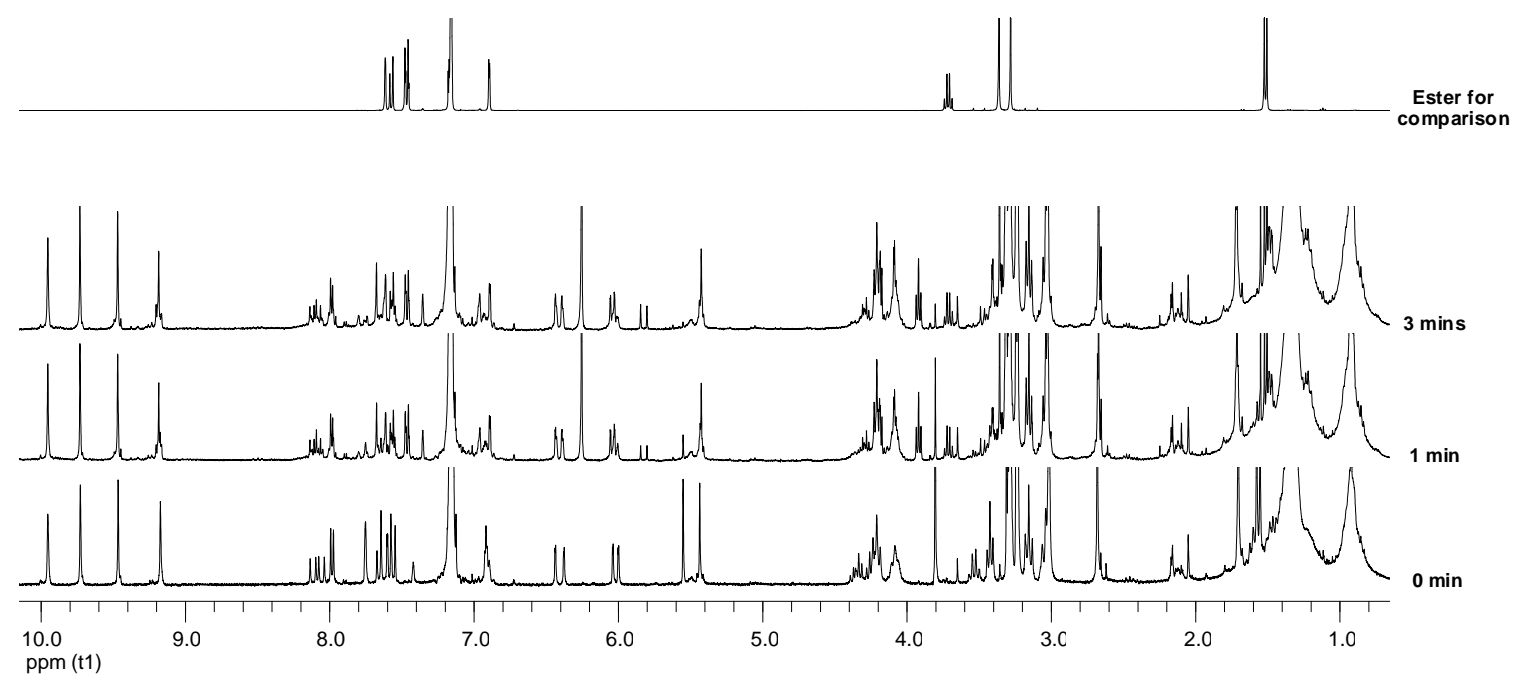

Figure 14 Photooxygenation of $\mathbf{8 Z}$ in $\mathrm{C}_{6} \mathrm{D}_{6}(400 \mathrm{MHz})$

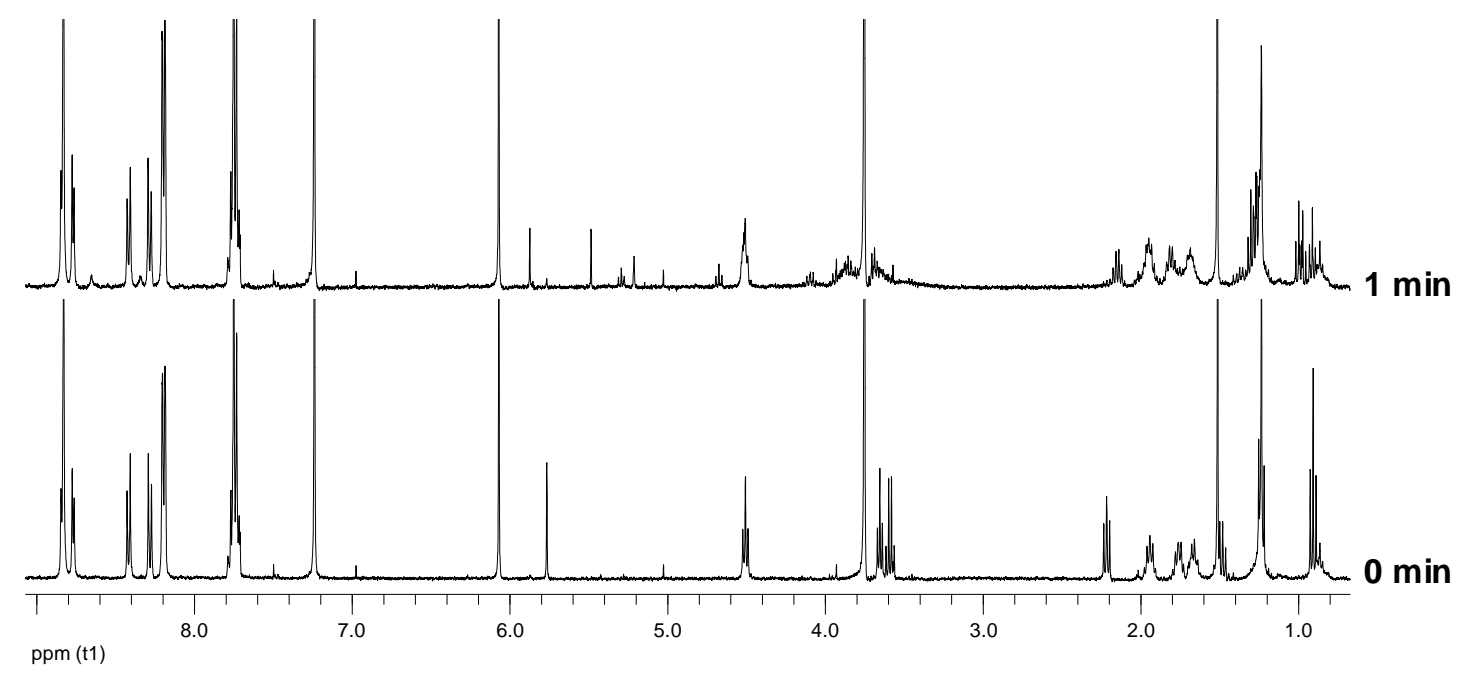

Figure 15 Photooxygenation of $2 \mathbf{E}$ in $\mathrm{CDCl}_{3}(400 \mathrm{MHz})$ 


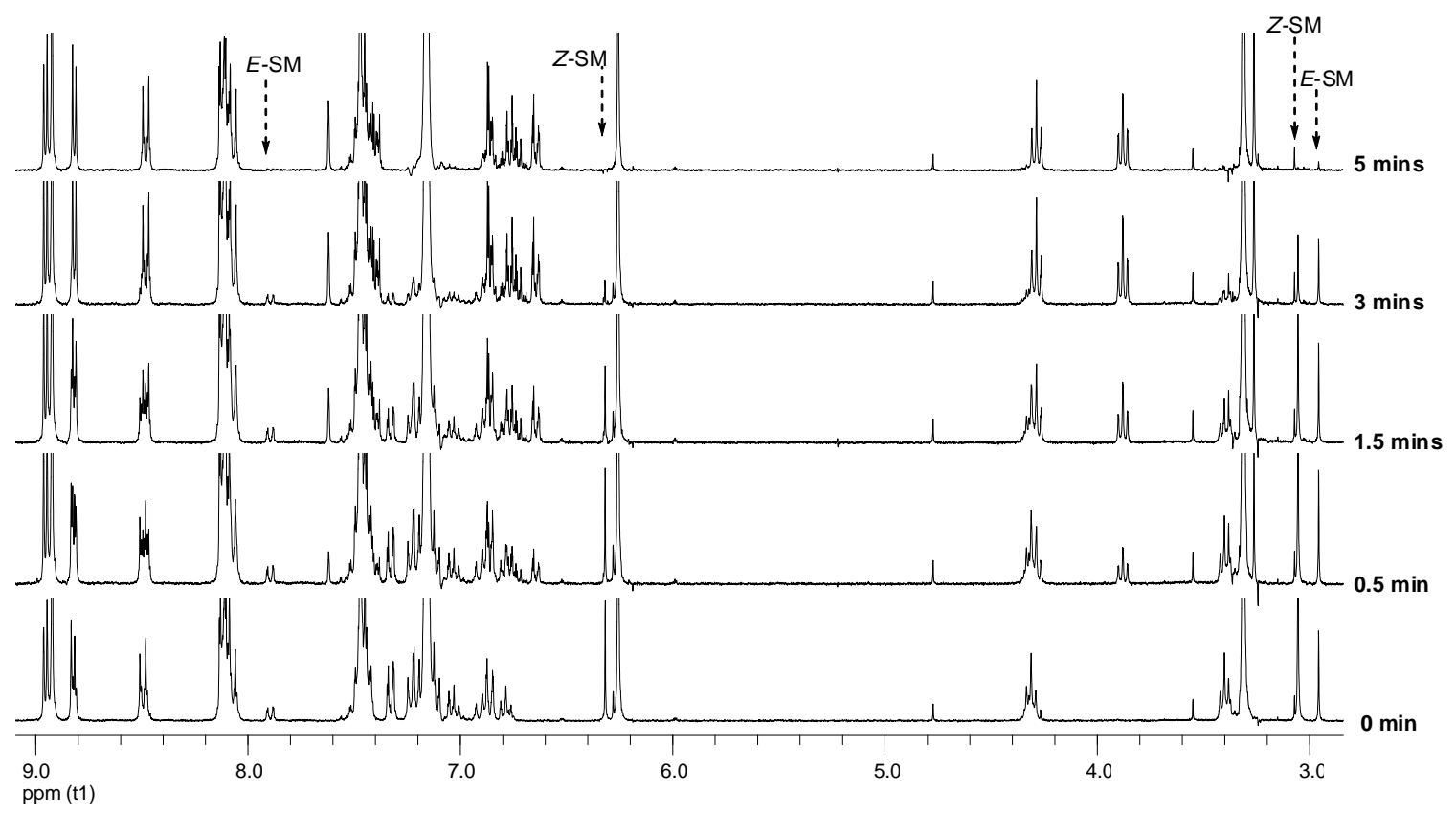

Figure 16 Photooxygenation of 9 in $\mathrm{C}_{6} \mathrm{D}_{6}(\mathrm{Z} / \mathrm{E}=4: 1,400 \mathrm{MHz})$ 


\section{References}

1. Perrin, D. D.; Armarego, W. L. F., Purification of Laboratory Chemicals. 3rd ed. ed.; Pergamon: 1988.

2. Takahashi, K.; Nakamura, H.; Furumoto, S.; Yamamoto, K.; Fukuda, H.; Matsumurae, A.; Yamamotoa, Y. Bioorgan. Med. Chem. 2005, (13), 735-43.

3. Takeda, T.; Sato, K.; Tsubouchi, A. Synthesis 2004, (9), 1457-65.

4. Koul, S.; Koul, J. L.; Singh, B.; Kapoor, M.; Parshad, R.; Manhas, K. S.; Taneja, S. C.; Qazi, G. N. Tetrahedron: Asymmetry 2005, (16), 2575-91.

5. $\quad$ Lee, D.-Y.; Hartwig, J. F. Org. Lett. 2005, 7, (6), 1169 -72.

6. Araki, N.; Obata, M.; Ichimura, A.; Mikata, Y.; Yano, S. Chem. Lett. 2004, 33, (4), 450-51.

7. Matile, S.; Berova, N.; Nakanishi, K.; Fleischhauer, J.; Woody, R. W. J. Am. Chem. Soc. 1996, 118, (22), 5198-206. 